\title{
Évaluation de processus : Programme Filles eveillées pour adolescentes migrantes employées de maison au Burkina Faso urbain 2012
}

Leah Jarvis

Gisele Kaboré

Population Council

Follow this and additional works at: https://knowledgecommons.popcouncil.org/departments_sbsr-pgy

Part of the Demography, Population, and Ecology Commons, Family, Life Course, and Society Commons, Gender and Sexuality Commons, International Public Health Commons, Medicine and Health Commons, Migration Studies Commons, and the Women's Health Commons How does access to this work benefit you? Let us know!

\section{Recommended Citation}

Jarvis, Leah and Gisele Kaboré. 2012. "Évaluation de processus : Programme Filles eveillées pour adolescentes migrantes employées de maison au Burkina Faso urbain 2012." Ouagadougou: Population Council. 
ÉVALUATION DE PROCESSUS:
PROGRAMME FILLES ÉVEILLÉES

POUR ADOLESCENTES MIGRANTES

EMPLOYÉES DE MAISON AU BURKINA

FASO URBAIN 2012

PREPARÉ PAR LEAH JARVIS

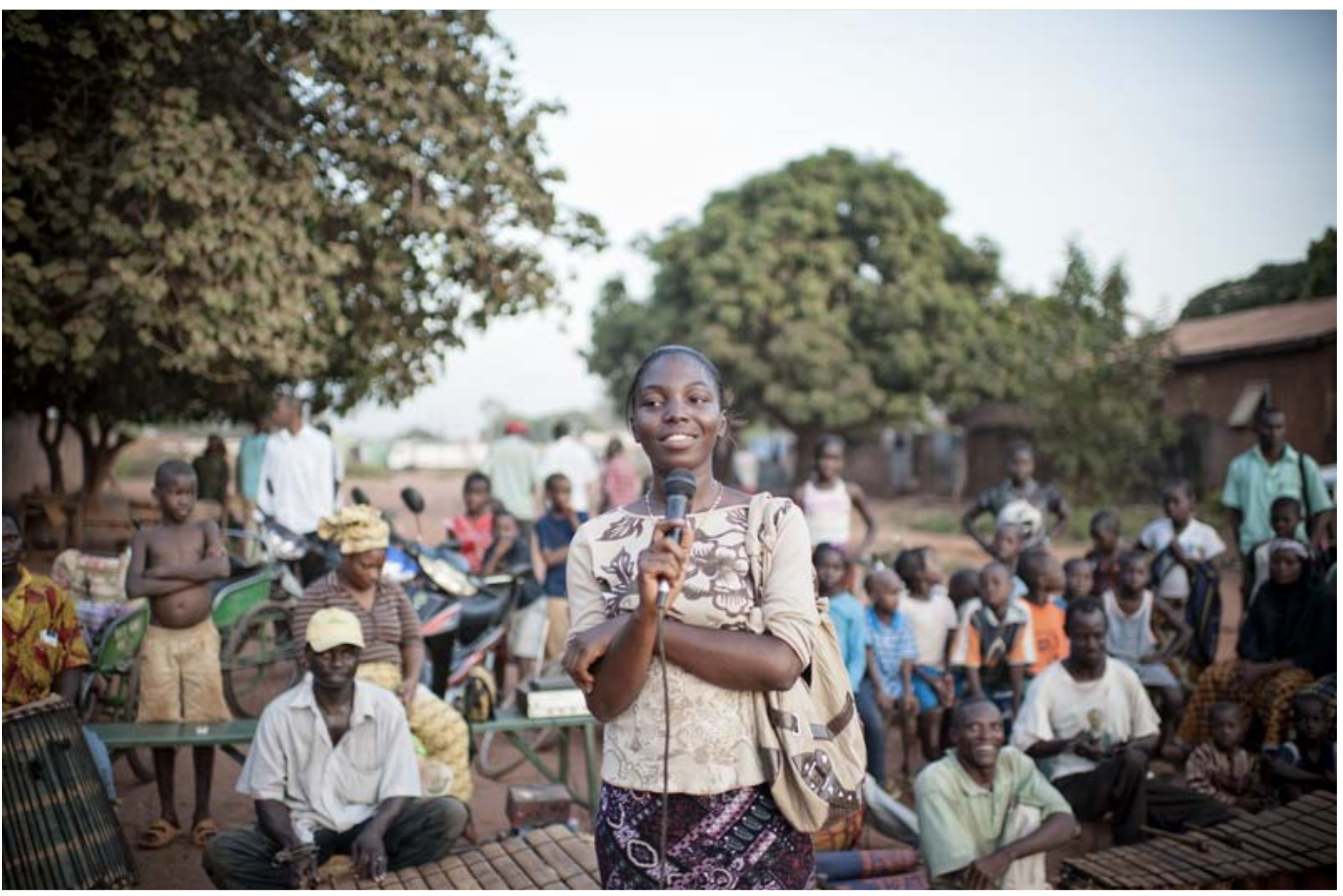




\section{(1) Population Council}

Le Population Council s'attelle aux questions cruciales de la santé et du développement - qu'il s'agisse d'endiguer la progression du VIH, d'améliorer la santé reproductive et d'offrir aux jeunes la promesse d'une vie satisfaisante et productive. Par ses travaux de recherche en biomédecine, en sciences sociales et en santé publique dans 50 pays, il s'efforce d'apporter avec ses partenaires des solutions qui conduisent à des politiques, des programmes et des technologies plus efficaces, pour améliorer la vie partout dans le monde. Fondé en 1952, le Population Council est une organisation non gouvernementale à but non lucratif. II siège à New York sous la conduite d'un conseil d'administration international.

Population Council

One Dag Hammarskjold Plaza

New York, NY 10017

Population Council

01 BP 6250

Ouagadougou 01

Burkina Faso

www.popcouncil.org

Indication de source suggérée: Leah Jarvis et Gisele Kabore. 2012. "Évaluation de processus: Programme Filles Éveillées pour adolescentes migrantes employées de maison au Burkina Faso urbain 2012." Ouagadougou: Population Council.

Photo courtoisement offerte par Ollivier Girard

(c) 2012 The Population Council, Inc. 


\section{Table des matières}

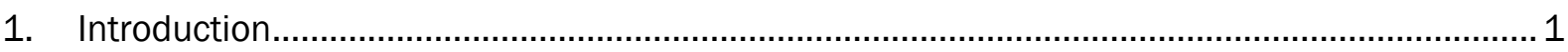

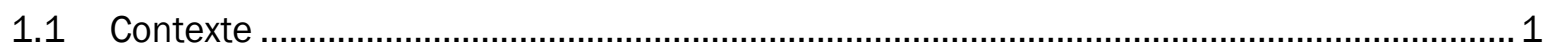

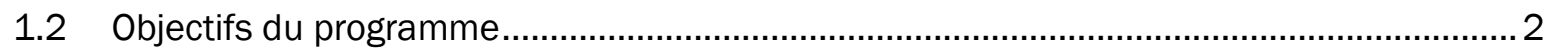

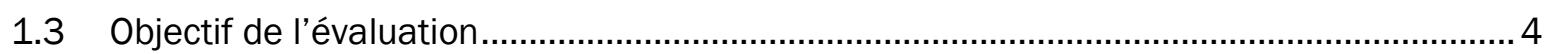

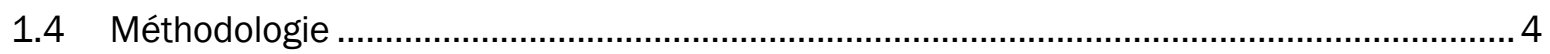

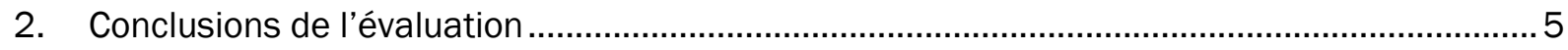

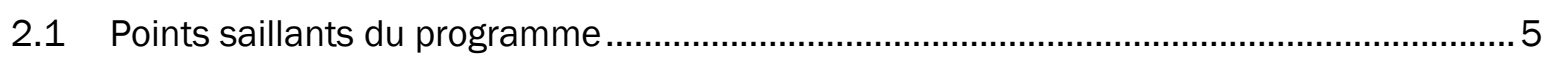

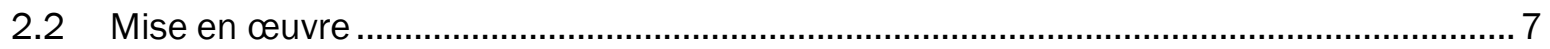

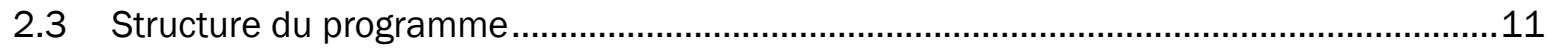

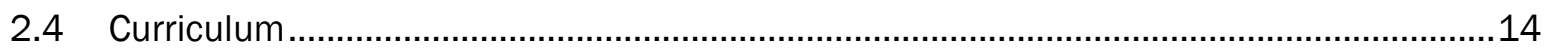

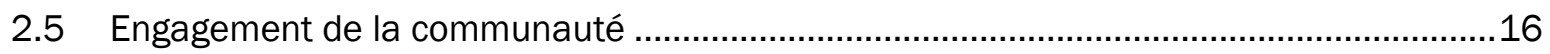

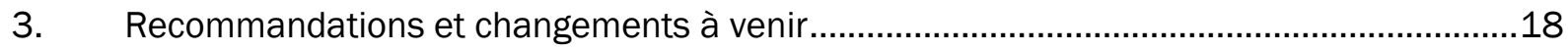

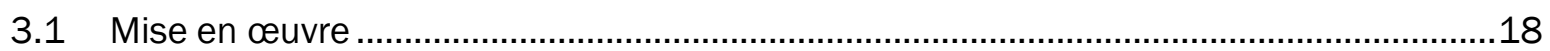

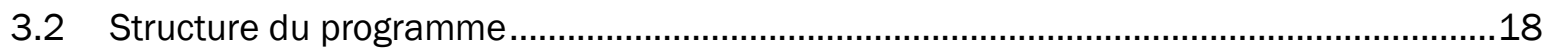

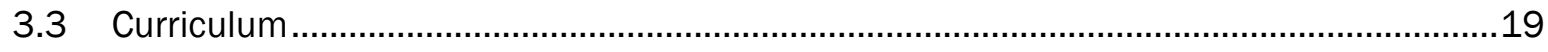

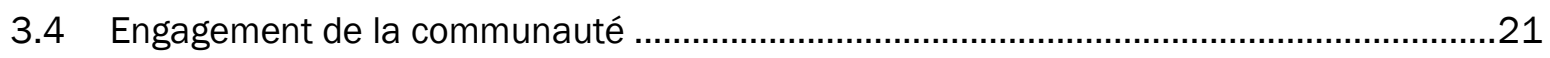

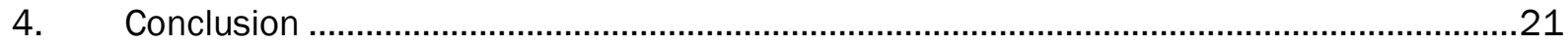

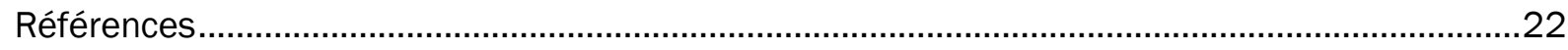

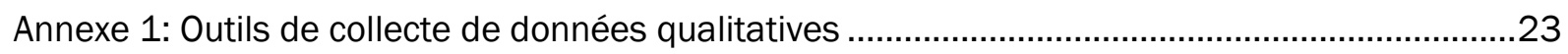

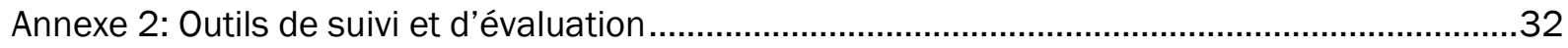




\section{INTRODUCTION}

Le Population Council est une organisation non gouvernementale vouée à la recherche en biomédecine, en sciences sociales et en santé publique, ainsi qu'à la dissémination de données qui conduisent à des politiques, des programmes et des technologies plus efficaces, pour améliorer la vie partout dans le monde. Le Council, qui travaille au Burkina Faso depuis 1989, a établi un bureau permanent dans le pays en 1994. Implanté dans 15 pays du monde, le Population Council a travaillé ou travaille actuellement dans plus de 50. Le programme Pauvreté, Genre et Jeunesse gère des projets de recherche et des programmes concernant les populations vulnérables telles que celle des adolescentes. Des programmes pilotes de renforcement des compétences axés sur les adolescentes vulnérables ont été menés avec succès en Inde, au Bangladesh, en Éthiopie, au Kenya, en Ouganda et au Guatemala, notamment, conduisant à une expansion de l'intervention dans presque tous ces pays (Population Council, 2012).

\subsection{Contexte}

Peuplé à $45,2 \%$ de jeunes de moins de 15 ans, le Burkina Faso abrite la 10e population la plus jeune du monde (Population Reference Bureau, 2011). Le pays se classe aussi parmi les plus faibles à l'indice de développement humain, en $181^{\mathrm{e}}$ place sur 188 (Programme des Nations Unies pour le Développement, 2011). Étant donné le haut taux de pauvreté et le grand nombre de jeunes, rares sont les débouchés économiques qui s'offrent à ces derniers, en particulier dans les milieux ruraux. La situation est pire encore pour les adolescentes, qui n'ont généralement bénéficié, au mieux, que d'une très faible scolarisation, formation ou autre préparation à l'emploi. L'impasse économique alimente la migration interne des jeunes, hommes et femmes, vers les milieux urbains, à la recherche de travail. Les jeunes femmes y deviennent souvent employées de maison. Certaines migrent seules; d'autres reçoivent l'assistance de parents ou sont accueillies à l'arrivée par des logeurs. ${ }^{1}$ Les adolescentes migrantes sont vulnérables aux mauvais traitements corporels et à l'exploitation économique. Elles courent aussi un risque accru de violence sexiste. Cela sans compter qu'elles travaillent de longues journées, vivent souvent avec leurs employeurs et sont généralement isolées, sans occasions de se faire des amies, de former de réseaux utiles ou de s'instruire. Comme l'indique la recherche, les interventions atteignent rarement, pour la plupart, les populations les plus vulnérables - les adolescentes notamment, en particulier celles déscolarisées et séparées de leur famille (Population Council; Guttmacher Institute, 2010). Souvent, ces populations ne reçoivent pas leur part équitable des services destinés aux jeunes.

À Ouagadougou, plus de 40\% des filles de 10 à 14 ans ne vivent ni avec leur père, ni avec leur mère ; $20 \%$ des filles de cette tranche d'âge ne sont en outre pas scolarisées, ce qui les rend particulièrement vulnérables et isolées (Population Council, 2009). II apparaît d'après la recherche formative du Population Council que beaucoup des filles migrent par étapes, d'abord vers une ville de moindre envergure telle que Bobo-Dioulasso où elles acquièrent l'expérience du service de maison avant de se rendre dans la capitale, à Ouagadougou. De grands nombres d'employées de maison regagnent chaque année leur village d'origine durant la saison des pluies

\footnotetext{
1 Les logeurs sont des familles qui ont déjà quitté les villages ruraux pour s'établir dans les villes. Lorsqu'elles arrivent à la ville, les filles migrantes prennent souvent contact avec un logeur originaire de leur village natal et s'installent avec sa famille en attendant de trouver un emploi.
} 
(de juin à septembre, généralement) pour aider aux travaux des champs. Les groupes d'âge et le calendrier du programme ont été établis en fonction de ces observations.

Bien qu'il existe déjà des programmes ciblés sur les adolescentes migrantes employées de maison à Bobo-Dioulasso et à Ouagadougou, la plupart restent étroitement axés sur l'atténuation des conséquences négatives après le fait (l'assistance aux victimes de violences sexuelles, par exemple), le perfectionnement professionnel ou l'information des filles au sujet de leurs droits légaux. Ces programmes ne sont du reste généralement pas conçus en fonction de segments d'âge logiques. La recherche du Population Council n'a guère révélé de programmes visant à renforcer l'aptitude des adolescentes migrantes employées de maison à se protéger. Filles éveillées recherche activement cette population de filles particulièrement vulnérables et entend réduire leur fragilité et élargir leurs horizons en les aidant à se préparer à une vie adulte productive.

\subsection{Objectifs du programme}

Le programme Filles éveillées a été conçu pour apporter aux adolescentes migrantes employées de maison les compétences, les connaissances et le capital social qui leur permettent de réduire leurs vulnérabilités et de s'ouvrir de nouveaux débouchés. Filles éveillées repose sur trois éléments clés d'un programme centré sur les filles: un espace de rencontre sûr, l'accès à des pairs de même sexe avec lesquelles elles peuvent établir un réseau social et l'assistance d'une femme mentor. Un "espace sûr " se définit comme un lieu où les jeunes filles peuvent se réunir et s'exprimer librement, sans crainte d'être entendues ou observées. Dans beaucoup de communautés, la majorité des espaces publics ou des centres de jeunesse sont dominés par les garçons et les hommes, dont la présence en fait des lieux non idéaux pour les jeunes filles. Les filles disposent rarement d'espaces sûrs, formels ou non, où elles puissent rencontrer leurs amies. Dans le contexte de Filles éveillées, l'espace sûr doit être aisément accessible aux filles (pas plus de 15 minutes à pied depuis la résidence ou le lieu de travail de chacune) et doit être disponible à l'usage exclusif des filles durant la période de leur rencontre hebdomadaire. Les mentors de Filles éveillées ont été sélectionnées en fonction de certains critères particuliers. Toutes devaient avoir entre 20 et 30 ans, pour être suffisamment âgées pour servir d'exemple aux participantes, mais suffisamment jeunes aussi pour que les filles se sentent à l'aise et puissent se confier à elles. Toutes devaient aussi avoir achevé pour le moins l'école primaire et elles devaient savoir lire et écrire. Les participantes à Filles éveillées devaient pour leur part être des adolescentes migrantes employées de maison, âgées de 11 à 16 ans dans la cohorte de Bobo-Dioulasso et de 15 à 18 ans pour celle de Ouagadougou.

Ces trois éléments clés d'un programme centré sur les filles aident ces dernières à renforcer leurs compétences et à améliorer leur capital social, tout en établissant le lien avec les services extérieurs. Filles éveillées est un programme mis au point par le Population Council sous financement de la Jabobs Foundation et mis en œuvre à travers les partenariats locaux établis au Burkina Faso, avec l'assistance technique du Population Council Burkina Faso et du siège de l'organisation à New York. À Bobo-Dioulasso, le partenaire à la mise en œuvre est l'Association Tié. À Ouagadougou, il s'agissait de l'Association Songui Manégré Aide au Développement Endogène (ASMADE) pour l'année 2011 et de l'Association d'Appui et d'Éveil Pugsada (ADEP) en 2012-2013. 
Grâce à un don consenti sur trois ans, le projet consiste en la mise en œuvre d'un curriculum de développement et renforcement de compétences appelé à atteindre un total de 300-400 adolescentes employées de maison à Ouagadougou et à Bobo-Dioulasso. Le programme compte deux cycles aux deux emplacements : le premier de novembre 2011 à juillet 2012 et le second de novembre 2012 à juin 2013. II est conçu pour s'organiser en sessions de groupe hebdomadaires d'une durée de 60 à 90 minutes chacune pendant huit mois, sous la conduite d'une mentor. Le calendrier du programme, de novembre à juin, coïncide avec la période pendant laquelle les filles vivent et travaillent dans les villes. Chaque cycle compte un total de 30 sessions, organisées en quatre modules : compétences de vie, capacités financières, santé et hygiène, et santé reproductive.

\section{Élaboration du curriculum}

Le curriculum des 30 sessions a été mis au point par le personnel du Population Council au Burkina et au siège de New York. II repose sur les conclusions de la recherche formative et s'inspire de programmes similaires existants, notamment Biruh Tesfa, organisé au bénéfice des adolescentes d'Éthiopie, et Adolescent Safe Spaces, Health, and Skills for Adulthood in Urban Ghana. Les quatre modules et 30 sessions ont été déterminés en fonction des besoins révélés par la recherche formative et des contraintes de temps de la population ciblée. Le programme a été rédigé en français, avec traduction des mots-clés en dioula et en moré, les deux langues principales parlées, respectivement, à Bobo-Dioulasso et à Ouagadougou. Au début de chaque session, les objectifs clairs que les participantes doivent pouvoir atteindre en fin de session sont définis et le programme est conçu de manière interactive pour encourager la participation et l'engagement. Le curriculum a été soumis aux organisations partenaires avant leur formation, afin d'obtenir leur feedback et de l'y incorporer. Chaque organisation a ensuite choisi une session par module pour un essai pilote auprès de filles admises de la communauté, suivi de l'incorporation du feedback obtenu à ce niveau. Une nouvelle version a été soumise et utilisée durant la formation des mentors, avec collecte et incorporation ultimes du feedback de ces dernières et des associations. Le curriculum a été finalisé en octobre 2011, avant le début des sessions hebdomadaires.

\section{Engagement de la communauté}

Filles éveillées comporte trois éléments spécifiquement conçus pour obtenir l'engagement de la communauté. Le premier consiste en une série de rencontres avec les leaders de la communauté. Les partenaires chargés de la mise en œuvre ont cherché à rencontrer ces leaders dans leurs districts respectifs afin de les informer du programme, de leur demander la permission d'utiliser des espaces de la communauté et d'obtenir leur appui. Deuxième élément, trois sessions de sensibilisation de la communauté ont été organisées durant le cycle en cours dans chaque quartier participant. Ces sessions ont pour objectif d'informer, de répondre aux questions de la communauté et d'obtenir son soutien. Enfin, le troisième élément prend la forme de visites à domicile, effectuées par les mentors aux résidences respectives des participantes. Les mentors sont censées effectuer au moins trois visites à l'employeur de chaque fille au cours des huit mois du programme, avec visites supplémentaires en cas de problèmes avec l'employeur ou exprimés par la fille, ou dans le cas d'absences répétées. Le but de ces visites à domicile est de renforcer la relation tant entre les mentors et les employeurs qu'entre ces derniers et les filles, ainsi que de résoudre les problèmes éventuels entre employeurs et employées et de tenir les employeurs au courant de l'évolution du programme et des progrès de leurs employées. 


\section{Branche facultative d'alphabétisation}

Bien que le Population Council reconnaisse l'importance de l'alphabétisation, la quantité de temps qui doit y être consacrée et celui limité dont dispose généralement la population ciblée rendent impraticable son intégration aux éléments centraux de Filles éveillées. Le programme s'était par conséquent proposé, dans un premier temps, d'offrir aux participantes l'occasion de s'inscrire à des cours d'alphabétisation en plus des sessions hebdomadaires. Les cours devaient leur apprendre à lire, à écrire et à calculer dans la langue locale (en dioula à Bobo-Dioulasso et en moré à Ouagadougou). Les filles ont toutefois exprimé une forte préférence pour l'apprentissage en français, qu'elles estiment plus utile à long terme. II a par conséquent été proposé aux filles intéressées de s'inscrire plutôt aux cours du soir. Ces cours sont organisés à l'intention des adolescents ou adultes qui n'ont jamais été scolarisés ou qui n'ont pas achevé leurs études, pour leur donner l'occasion d'obtenir une instruction primaire ou secondaire, à partir de n'importe quel niveau.

\section{Formation au programme}

En préparation au programme, le Population Council a organisé une formation à l'intention des partenaires de mise en œuvre, ensuite appelés à former à leur tour les mentors. Ces dernières ont ensuite recruté les participantes dans leurs quartiers respectifs.

Le premier cycle s'est déroulé de novembre 2011 à juin 2012 ; le second courra de novembre 2012 à juin 2013. Le premier cycle visait un minimum de 60 à 70 filles à Bobo-Dioulasso et 100 filles à Ouagadougou. Les mentors de Bobo-Dioulasso ont recruté 90 filles (30 dans chacun de trois groupes) et celles de Ouagadougou en ont recruté 150 (30 dans chacun de cinq groupes), pour compenser l'attrition et la mobilité.

\subsection{Objectif de l'évaluation}

L'évaluation de processus cherche à évaluer différents aspects du programme Filles éveillées, dans le but de l'améliorer pour le second cycle, à partir de novembre 2012. Plus spécifiquement, le but est d'évaluer:

- les éléments du programme qui ont été mis en œuvre ou qui ne l'ont pas été comme planifié et les obstacles à la mise en œuvre; et

- les améliorations pouvant être apportées à la structure, la mise en œuvre et le contenu programmatique, afin de réduire plus efficacement les vulnérabilités de la population ciblée et d'élargir ses horizons.

\subsection{Méthodologie}

La méthodologie adoptée pour cette évaluation aura été la collecte de données qualitatives primaires, du 9 au 12 juillet 2012, et l'analyse soignée des données qualitatives et quantitatives primaires collectées au fil du programme à l'aide d'outils de suivi et d'évaluation. Une stagiaire et la coordinatrice du programme pour le Population Council au Burkina ont été chargées de la rédaction de guides de discussion de groupe et d'entretien en profondeur. Des discussions de groupe et des entretiens en profondeur ont été menés en dioula, puis transcrits en français par un consultant indépendant. Les guides de discussion de groupe et d'entretien sont joints pour référence à l'Annexe 1. Une série d'outils de suivi et d'évaluation, créés par le Population Council avant le lancement du programme, ont été remplis par les mentors et les organisations 
partenaires en cours de déroulement du programme (voir l'Annexe 2). Ces outils ont été examinés aux fins de cette évaluation.

Outils de suivi et d'évaluation employés :

i. Rapports des activités et sessions remplis par les mentors :

a. Rapports d'activité remplis par les mentors à chaque session ("Canevas d'appréciation des session ")

b. Données de présence à chaque session ("Tableau de présence des filles aux sessions hebdomadaires")

c. Formulaires individuels concernant les visites à domicile ("Fiche individuelle des visites à domicile ")

ii. Fiches de supervision des mentors remplies par l'Association Tié ("Fiche de supervision des activités des mentors")

iii. Trois rapports trimestriels soumis au Population Council par l'Association Tié suivant les directives applicables aux rapports ("Canevas de rapport narratif des associations ")

iv. Rapport de progression des élèves rempli par les enseignants des cours du soir ("Canevas du rapport des enseignants sur l'encadrement des filles du programme)

v. Feedback sur le curriculum recueilli par l'Association Tié, partenaire de mise en œuvre à Bobo-Dioulasso

Données qualitatives primaires collectées :

i. Deux discussions de groupe avec les participantes au programme (un groupe de 15 filles - Secteur 9/10 et un groupe de neuf filles - Secteur 2)

ii. Deux discussions de groupe avec les membres de la communauté (un groupe de sept femmes et un groupe de cinq hommes)

iii. Une discussion de groupe avec les trois membres de l'Association Tié responsables du programme Filles éveillées

iv. Deux discussions de groupe avec les mentors du programme (trois dans chaque groupe)

v. Trois entretiens en profondeur avec les employeurs

vi. Entretien informel avec la coordinatrice du Population Council

\section{CONCLUSIONS DE L'EVALUATION}

Les résultats de cette évaluation sont organisés en cinq grandes catégories : points saillants du programme, mise en œuvre, structure, contenu et curriculum.

\subsection{Points saillants du programme}

Dans l'ensemble, les discussions de groupe et les entretiens en profondeur ont révélé une très bonne acceptation du programme Filles éveillées, largement apprécié par les employeurs, les membres de la communauté et les participantes. Toutes les personnes interviewées ont fait l'éloge de l'Association Tié, du Population Council et des mentors pour leur travail et pour l'élaboration du programme. Tous les employeurs interviewés ont déclaré qu'ils recommanderaient certainement le programme à d'autres et qu'ils laisseraient d'autres employées de maison y participer dans le futur. Les employeurs et les membres masculins 
et féminins de la communauté ont souligné qu'après leur participation à Filles éveillées, les filles se sont montrées plus respectueuses, ont fait preuve d'une plus grande stabilité (moins susceptibles de changer fréquemment d'employeur), d'une meilleure hygiène personnelle et qu'elles nettoyaient mieux. Les membres féminines de la communauté ont fait remarquer que les employeurs sont moins susceptibles de maltraiter les filles ou de ne pas les payer régulièrement. Les employeurs ont dit apprécier le fait que les filles avaient appris à épargner, à lire et à écrire, et que le programme avait couvert des thèmes, comme celui de la sexualité, qu'ils n'aborderaient pas eux-mêmes avec leurs employées. Plusieurs employeurs et membres de la communauté ont également mentionné les avantages de la disposition des participantes à partager les connaissances apprises, tant avec les enfants du ménage de l'employeur qu'avec d'autres membres de leurs villages d'origine. Les mentors se sont réjouies de l'occasion données aux filles de se faire des amies, une option qui ne leur est sinon pas souvent offerte. Enfin, les participantes elles-mêmes ont cité, parmi leurs aspects préférés du programme, l'apprentissage concernant l'épargne, leur protection contre la grossesse et le $\mathrm{VIH}$ et la scolarisation.

L'élément le plus souvent cité comme différenciant ce programme par rapport à d'autres concerne l'implication de la communauté. Les membres de la communauté, les mentors et les employeurs ont tous apprécié les efforts du programme à chercher le soutien des employeurs et des leaders de la communauté à travers les sessions communautaires, les visites à domicile et les formulaires de consentement des employeurs, plutôt que de recruter directement les adolescentes. Cet aspect du programme a été mentionné à maintes reprises et se distingue tel un élément extrêmement positif. L'Association Tié a également souligné avoir apprécié l'approche méthodique bien structurée du Population Council, ainsi que la nature interactive du curriculum.

« Avant le programme, nous ne savions rien du sida, du cancer et de la blennorragie. Ce qui m'a vraiment plu dans le programme, c'est ce que nous avons appris sur ces maladies. »

- Participante de 12 ans

«Un jour [mon employée] m'a dit qu'avant, les femmes étaient en position de faiblesse, mais aujourd'hui, la femme a les mêmes droits que l'homme. Si elle étudie bien, elle pourra devenir l'égale d'un homme. Cela m'a vraiment frappé et est resté marqué dans mon cerveau. » - Employeuse

«Le programme est vraiment bénéfique aux filles. Elles ont changé physiquement, dans leur manière de parler et d'écouter. »

- Membre féminine de la communauté

« Nous, les mères, ne nous sentons vraiment pas à l'aise à l'idée de parler de sexualité avec nos filles. Quand elles vont aux sessions, elles apprennent beaucoup et je m'en réjouis. Nous ne les informons jamais sur la menstruation. »

- Employeuse

«Grâce au programme, les employeurs prennent les choses au sérieux et le salaire des filles est régulier. »

- Membre féminine de la communauté 


\subsection{Mise en œuvre}

\section{Formation des partenariats}

L'un des objectifs de la recherche formative menée en 2010 était d'identifier les organisations qui travaillaient déjà avec la population ciblée à Ouagadougou et à Bobo-Dioulasso et de sélectionner ainsi des partenaires pour cette intervention. À Bobo-Dioulasso, l'Association Tié a été sélectionnée comme partenaire potentielle, tandis que les organisations ADEP et ASMADE étaient identifiées à ce titre à Ouagadougou. Les négociations contractuelles avec l'Association Tié et avec ASMADE ont été entamées en 2011.

\section{Formation des partenaires}

En septembre 2011, le Population Council a organisé une formation de quatre jours à l'intention de l'Association Tié et d'ASMADE. Trois employés de chaque organisation y ont participé. La formation a couvert les caractéristiques sociodémographiques de la population ciblée, la raison du choix de cette population et de l'intervention, les objectifs poursuivis, les tâches requises de la part des partenaires à la mise en œuvre, les difficultés prévisibles et un examen approfondi du curriculum.

D'après le feedback reçu des membres de l'Association Tié, quatre jours ne suffisent pas à cette formation. Les

« Je pense que la formation était vraiment pertinente. Nous avons appris énormément, le programme était extrêmement riche, mais force est de reconnaître que la durée prévue était insuffisante. Nous n'avons pu développer certains éléments qu'à un niveau superficiel. » - Employée de l’Association Tié participants ont apprécié le contenu de la formation et la transmission de connaissances importantes concernant la vulnérabilité des filles et de la population ciblée en particulier. Ils ont toutefois suggéré une formation de plus longue durée, avec plus de temps consacré à la compréhension de la structure du programme avant de passer aux modules du curriculum en soi. L'Association Tié a également indiqué qu'il serait utile de consacrer plus de temps à la discussion de la traduction des mots et concepts clés du français en dioula et en moré.

\section{Partenaire de Ouagadougou}

Invitée à s'associer au titre de partenaire au programme Filles éveillées, l'organisation ASMADE s'est dite intéressée et disponible. Un contrat a été négocié et signé. En septembre et octobre 2011, ASMADE a assumé ses fonctions. Elle a assisté à la formation des partenaires, sélectionné les secteurs d'organisation des groupes, recruté ses mentors et organisé leur formation. Les mentors ont recruté les participantes au programme et les données de base destinées à l'étude quantitative ont été collectées auprès de celles-ci. En novembre, ASMADE a cependant indiqué ne pas disposer d'un temps-effectifs suffisant à la gestion du projet et suggéré l'engagement d'effectifs supplémentaires. Le Population Council a accepté la demande, le budget a été révisé et une personne supplémentaire a été engagée au service d'ASMADE. D'autres problèmes sont cependant apparus et, en décembre 2011, après de sérieuses négociations et délibérations, la décision mutuelle a été prise qu'ASMADE ne serait pas en mesure d'assurer la mise en œuvre de Filles éveillées. Aucune session n'a par conséquent eu lieu pour les groupes de filles de Ouagadougou. 


\section{Ajustements au programme de Bobo-Dioulasso}

En raison de la date tardive du retrait d'ASMADE à Ouagadougou, la recherche d'une organisation de remplacement, la négociation d'un nouveau contrat et la formation de la nouvelle organisation auraient par trop différé la mise en œuvre des groupes planifiés. Aussi le Population Council a-t-il plutôt décidé d'élargir le contrat conclu avec l'Association Tié de manière à recruter trois autres groupes de filles et trois autres mentors dans trois autres secteurs de Bobo-Dioulasso. L'idée a été soumise à l'Association Tié et à la Jabobs Foundation. Toutes les parties ont convenu qu'il s'agissait là de la meilleure solution. Le recrutement des nouvelles mentors a été suivi d'une formation de trois jours et du lancement du programme dans les trois nouvelles communautés. Afin d'éviter tous autres délais, certaines mentors ont entamé les sessions avant que ne soient recrutées les 30 participantes à chaque groupe. L'Association Tié avait initialement exploré l'idée de tenir deux sessions par semaine (une le dimanche et une le jeudi) pour rattraper le temps perdu, mais il est apparu que les employeurs n'étaient pas disposés à autoriser la participation de leurs employées à plus d'une session hebdomadaire. Il a donc été décidé de couvrir deux leçons chaque dimanche, pour assurer l'achèvement du programme en l'espace de 15 semaines, à concurrence toutefois de sessions prolongées sur deux à trois heures. L'approche n'a pas manqué de présenter ses propres difficultés et les mentors ont souligné la tendance des filles à se montrer fatiguées, distraites et moins engagées lors de la seconde leçon. Étant donné les circonstances toutefois, la solution s'est avérée satisfaisante pour tous les intéressés et a permis au nombre de filles initialement ciblé de participer au programme.

\section{Recrutement et formation des mentors}

L'Association Tié aurait aimé disposer de plus de temps entre la formation de ses représentants et le lancement du programme, pour le recrutement et la formation des mentors. Pour l'assister dans ce recrutement, l'Association Tié a engagé les services d'un annonceur public ayant annoncé les postes proposés dans les secteurs pertinents au moyen d'un haut-parleur monté sur une voiture. L'organisation a invité les candidates répondant aux critères d'âge et d'éducation à soumettre leur candidature et leur C.V. et à se présenter à une entrevue. Six candidates se sont présentées et trois mentors plus une remplaçante éventuelle ont été sélectionnées. L'Association Tié a pu engager et former les mentors conformément au calendrier fixé mais elle a déclaré s'être sentie pressée.

Avant de se lancer dans le recrutement des participantes, les mentors de Bobo-Dioulasso ont elles-mêmes reçu une formation de trois jours de l'Association Tié, axée sur les objectifs du programme, les exigences relatives aux visites à domicile (VAD), les réunions communautaires, les outils d'évaluation et le passage en revue de chaque leçon du curriculum. Deux formations distinctes ont été organisées : la première en octobre 2011 pour les mentors des secteurs 2, 4 et 9/10, et la seconde en mars 2012 pour celles des secteurs 8,17 et 20 . Les mentors ont déclaré unanimement que si le contenu de la formation était très bon, la formation en soi était trop courte. Elles ont exprimé le désir d'approfondir davantage le curriculum, d'apprendre les techniques d'enseignement et de mobilisation et de discuter l'explication des concepts clés en dioula. Les mentors ont suggéré au moins une journée de formation supplémentaire. 
Relation entre les mentors et l'Association Tié

Dans l'ensemble, la relation entre les mentors et l'Association Tié a été qualifiée de très positive. Les mentors ont déclaré que l'Association Tié les avaient soutenues lorsque nécessaire dans la résolution des différends entre les employeurs et les participantes, les accompagnant parfois lors des visites à domicile. Les mentors ont également qualifié d'utiles les réunions hebdomadaires tenues après chaque session avec l'Association Tié, déclarant aussi que l'association les avait aidées à se préparer aux sessions suivantes,

«Si l'employeur veut se désister pour que les filles n'assistent plus au programme et si nous intervenons mais que cela ne sert à rien, nous appelons l'Association : elle envoie quelqu'un et le problème est résolu.

En général, quand l'Association intervient, tout rentre dans l'ordre. »

- Mentor du programme, Bobo-

Dioulasso concernant surtout les questions de présentation du curriculum en dioula.

Un incident particulier aurait cependant dû, de l'avis de la mentor concernée, recevoir une réponse plus rapide et attentive de la part de l'Association Tié. La mentor en question avait reçu un appel, après une session, l'informant qu'une des participante employée de maison n'était pas rentrée chez elle. II s'en était suivi plusieurs heures de recherche de la fille, qui avait finalement été retrouvée saine et sauve. Avec une autre participante au programme, la fille avait décidé de s'enfuir à Ouagadougou, mais l'idée avait mal tourné et la fille s'était retrouvée dans la rue pour la nuit. L'employeur avait d'abord accusé la mentor d'avoir incité la fille à s'enfuir, mais celle-ci avait fini par avouer que l'idée était venue de son amie et non de sa mentor. Bien que la situation ait été résolue, la mentor a exprimé son mécontentement face au fait qu'elle avait essayé de joindre à plusieurs reprises les membres de l'Association Tié mais que personne n'avait répondu avant le lendemain matin. Comme les sessions se tiennent le dimanche, il est important que les mentors puissent joindre le personnel chargé du programme pendant le week-end et que celui-ci leur réponde sans tarder et de manière appropriée.

\section{Visites à domicile (VAD)}

Les mentors étaient censées effectuer au moins trois visites au domicile de l'employeur de chaque participante durant les huit mois du programme, avec visites supplémentaires en cas d'incidents particuliers ou d'absences répétées. Compte tenu de l'attrition des participantes et du fait que quelques filles avaient le même employeur, chaque mentor aurait dû effectuer un minimum de 75 à 90 VAD.

Le nombre de VAD effectuées varie grandement d'une mentor à l'autre, deux d'entre elles n'en ayant effectué que 17 chacune tandis qu'une autre les avait effectuées toutes les 90. Les difficultés associées aux VAD concernent l'absence ou l'indisponibilité des employées, sans justifier toutefois de si faibles nombres pour certaines mentors. Dans l'ensemble, les VAD se sont avérées un outil précieux d'encouragement des employeurs à soutenir le programme. Les mentors comme les
« [Les VAD] ont été utiles, pour nous comme pour nos employeurs, parce ce que le programme nous était bénéfique et que cela profitait à nos employeurs. Ils ont vu l'importance du programme et ils nous ont laissé participer. »

- Participante de 16 ans

« Nos rapports avec nos employeurs ont changé. Au début, ils étaient réticents, mais après les VAD, ils ont compris et nous ont

laissé participer au programme. » - Participante de 16 ans 
participantes ont déclaré que les VAD encourageaient les employeurs à autoriser les filles à interrompre leur travail et à arriver aux sessions à temps. D'après ce qu'en ont dit les employeurs, les VAD les ont aidés à prendre le programme plus au sérieux et à se tenir au courant de ce que leurs employées y apprenaient. Les mentors ont également indiqué que les VAD ont, à plus d'une reprise, facilité la résolution d'un conflit entre l'employée et son employeur. Dans les cas où les mentors n'avaient pas réussi à résoudre un conflit, les membres de I'Association Tié les avaient accompagnées dans leurs VAD - geste dans lequel les mentors ont vu l'un des types de soutien les plus utiles apportés par Tié.

\section{Espaces sûrs et logistique du programme}

Dans l'ensemble, l'Association Tié et les mentors ont qualifié le concept des espaces sûrs d'utile et productif. Des accords verbaux avaient été conclus avec la communauté et/ou les responsables de l'espace pour assurer son accessibilité pendant toute la durée du programme. Les espaces sûrs ont été recherchés et sélectionnés par l'organisation chargée de la mise en œuvre. Leur usage a été négocié avec les leaders de la communauté et/ou les propriétaires de l'espace. Les églises, mosquées et autres lieux de culte ont été exclus pour éviter l'association du programme avec une religion et l'aliénation potentielle résultante de certaines participantes. À Bobo-Dioulasso, deux espaces sûrs ont été établis dans des écoles primaires et un dans une garderie préscolaire. Un autre espace a été négocié dans une salle de réunion d'un établissement occupé par le ministère de l'Action sociale et un cinquième dans l'espace d'une ONG locale vouée à l'enfance vulnérable. Le dernier espace obtenu l'a été dans une cour de séchage de mangues à vendre au marché. Trois de ces espaces ont nécessité la fabrication de bancs ; les trois autres disposaient déjà de sièges adéquats. Trois étaient des locaux fermés ; les trois autres, des espaces couverts d'un toit mais sans parois. Certains problèmes logistiques se sont posés suivant l'espace utilisé. Par exemple, les espaces ouverts ont présenté des problèmes par temps de pluie ou de vent (difficulté d'entendre ce qui était dit et filles mouillées par la pluie). La mentor du secteur 20 a déclaré que son espace, la cour destinée au séchage des mangues, était trop isolé. Pour plusieurs leçons, il aurait aussi fallu afficher des feuilles de papier au mur, opération impossible dans certains des espaces.

Les mentors ont aussi mentionné que pour les exercices requérant l'utilisation de billets et de pièces de monnaie (francs CFA, monnaie de I'Union Économique et Monétaire Ouest-Africaine en circulation au Burkina Faso) dans la section consacrée aux capacités financières, elles ne disposaient pas de l'argent nécessaire. Le curriculum précise l'usage de faux billets, mais leur production est illégale au Burkina Faso et ils ne seraient du reste pas très utiles étant donné que la majorité des filles sont analphabètes. L'usage de vrais billets et pièces serait plus approprié, car même les participantes analphabètes peuvent en reconnaître la valeur d'après la couleur et la taille. On leur apporterait ainsi une connaissance plus directement applicable. 


\subsection{Structure du programme}

\section{Programmation des sessions}

Le jour et l'heure choisis pour les rencontres sont le produit de la recherche formative. Ils ont été choisis pour coïncider avec le jour de la semaine où le plus grand nombre de participantes pourrait y assister, pendant les mois où la majorité des filles vivent en ville. Certains employeurs ont estimé le dimanche un jour difficile en raison de la quantité de travail à accomplir le weekend. Pour la plupart, le dimanche après-midi reste cependant le meilleur moment.

Beaucoup de participantes ont jugé trop courte la durée de huit mois. Parmi celles inscrites au programme accéléré, certaines ont indiqué qu'elles désireraient répéter leur participation. La raison n'en était, pour aucune fille toutefois, un manque de compréhension des leçons. La motivation était plutôt que les filles aimaient le programme et qu'elles désiraient y participer plus longtemps. La mobilité de la population ciblée rend impossible cette prolongation, mais il est encourageant de savoir que les participantes ont apprécié le programme au point de désirer s'y réinscrire ou le poursuivre. En 2012, la saison des pluies s'est déclarée un peu plus tôt que d'habitude, amenant de nombreuses filles migrantes à regagner leur village d'origine plus tôt que les autres années. De plus, le calendrier du programme a fait que les filles inscrites aux cours du soir ont manqué le début de ces classes (voir plus loin).

\section{Présences}

En moyenne, les participantes à Filles éveillées ont assisté à 22 des 30 sessions. Des 178 participantes au programme, 128 (72\%) ont assisté à au moins 20 des sessions, y compris 33 filles $(19 \%)$ présentes à chaque session. Les principaux obstacles à la présence régulière des filles se sont avérés la quantité de travail à accomplir chez l'employeur ou les tâches demandées par les employeurs juste avant l'heure prévue de la session. Les mentors ont effectué de nombreuses VAD afin de déterminer la raison de l'absence des filles et d'essayer de sensibiliser les employeurs à l'importance de leur présence à chaque session. Parmi les autres raisons découvertes, on retiendra la maladie et les événements ou urgences dans la famille de l'employeur, tels qu'un mariage ou un décès. Dans certains cas, les mentors n'ont pas pu identifier la raison de l'absence.

Un autre problème est apparu dans l'arrivée tardive des filles et dans l'interruption des sessions ainsi causée, exigeant parfois la réexplication de concepts déjà présentés aux autres. Toutes les mentors ont déclaré que quelques filles au moins arrivaient toujours en retard à chaque session. Les raisons en étaient la quantité de travail à accomplir et les tâches demandées par les employeurs juste avant l'heure prévue de la session.

Les raisons d'attrition se sont révélées la quantité de travail ou les restrictions imposés par l'employeur, le retour au village d'origine ou, dans le cas d'une fille, la naissance d'un enfant. Quarante-sept filles ont commencé le programme mais l'ont abandonné au moins un mois avant la fin des sessions, y compris 31 vers la moitié ou plus tôt. 


\section{Mentors}

Les participantes ont qualifié de très positives leurs relations avec leur mentor. Durant les discussions de groupe, elles ont dit considérer leur mentor comme une amie, lui demander conseil et ne pas hésiter à lui poser des questions. Les mentors ont également déclaré que les participantes faisaient appel à elles pour leur demander conseil sur différents sujets, comme par exemple le mariage et les relations avec leurs employeurs. Les participantes ont indiqué, parmi ses qualités les plus importantes, qu'une bonne mentor est discrète, digne de confiance, attentive, polie et accueillante.
«Quand j'ai commencé à travailler, au début, je me disputais souvent avec mon employeur et je voulais quitter mon emploi, mais grâce à ma mentor, j'ai décidé de rester. Elle m'a donné de bons conseils. »

- Participante de 16 ans

«On vient parce qu'on se sent à l'aise avec [notre mentor] et qu'on peut lui dire

ce qu'on pense. Avec elle, on n'a pas peur de communiquer - on la considère comme une amie. »

- Participante de 15 ans

\section{Amitiés des participantes}

Les mentors, les participantes et l'Association Tié ont toutes fait remarquer que les participantes de chaque secteur avaient noué de solides amitiés et qu'il s'agissait là d'un bienfait attribuable au programme. Et les mentors d'ajouter que les adolescentes migrantes employées de maison n'ont sinon pas l'occasion de se faire des amies au travail. Les participantes elles-mêmes ont indiqué que leur groupe était devenu comme une famille.

«Si une fille est malade ou qu'elle a perdu un parent, on lui rend visite. »

- Participante de 14 ans

"S'il y a un mariage ou un baptême, on se cotise toutes et on donne quelque chose à la personne. On est comme une famille. »

- Participante de 15 ans

\section{Groupes d'âge ciblés}

Pour les trois premiers groupes, dans les secteurs 2, 4 et 9/10, les participantes étaient âgées de 11 à 16 ans. Dans leur feedback régulier, les mentors ont indiqué qu'il était parfois difficile pour les plus jeunes de saisir le sens des leçons et de comprendre leurs aînées concernant les sujets tels que la sexualité. Aussi le Population Council a-t-il décidé de faire l'expérience d'une répartition différente des âges dans les quatrième, cinquième et sixième groupes, dans les secteurs 8, 17 et 20. La tranche d'âge est moindre, de 14 à 16 ans dans deux groupes et de 11 à 13 ans dans le troisième. La mentor du groupe le plus jeune, de 11 à 13 ans, a cependant fait état de difficultés similaires en termes de compréhension et d'engagement des cadettes. La séparation des plus jeunes ne semble pas avoir aidé à remédier au problème. L'Association Tié a également déclaré que l'identification et le recrutement d'un nombre suffisant de filles dans ces groupes d'âge plus spécifiques avaient été difficiles, surtout en ce qui concerne les plus jeunes (11 à 13 ans). Les opinions des mentors diffèrent quant à savoir s'il convient ou non de diviser les groupes en fonction de l'âge.

\section{Nourriture}

L'offre d'un repas aux participantes à la fin de chaque session s'est avérée une partie importante du programme. D'autres programmes pilotes comparables du Population Council, menés dans d'autres contextes, n'ont pas adopté cette approche. La recherche formative a cependant indiqué que pratiquement tous les programmes visant la même population au Burkina offrent de la nourriture. Étant donné le niveau de pauvreté, les filles n'ont souvent aucun autre moyen de se 
procurer un repas complet le jour des sessions, surtout si leur participation leur fait manquer un repas au domicile de leur employeur. II a par conséquent été jugé important de nourrir les filles dans le cadre du programme.

\section{Clôture du programme}

Durant la première année du programme, aucune fête ou cérémonie n'a été planifiée pour reconnaître l'accomplissement des participantes. Plusieurs filles en ont été déçues et se sont plaintes de la terminaison abrupte du programme. Mentors, participantes et employeurs ont tous suggéré l'organisation d'une cérémonie ou d'une fête pour marquer la fin du programme.

\section{Cours d'alphabétisation et cours du soir}

Sur les 90 filles inscrites aux trois premiers groupes de Filles éveillées, 59 se sont inscrites aussi aux cours du soir. Leurs droits d'inscription et frais ont été couverts par le programme Filles éveillées, sous financement du Population Council. De ces filles, 39 se sont inscrites au niveau élémentaire, soit l'équivalent de la première primaire. Quarante-deux filles (71\%) ont obtenu des notes adéquates pour passer au niveau suivant, tandis que 11 ont abandonné les cours du soir. Pour l'ensemble, la note globale s'est avérée de 5,12/10.

La justification initiale de l'offre de cours d'alphabétisation, par opposition aux cours du soir, était que les participantes puissent achever les cours proposés pendant la durée du programme, alors que les cours du soir exigent plusieurs années pour ceux

« Avant, je n'avais jamais été à l'école mais, grâce au programme, j'ai appris à lire et à écrire. » - Participante de 16 ans

qui commencent au niveau élémentaire. Les filles qui suivent les cours du soir et qui y obtiennent de bonnes notes désirent poursuivre leur éducation mais ne le pourraient pas faute d'un financement continu de la part du Population Council. La décision a été prise de continuer à payer les frais scolaires des filles qui ont participé à Filles éveillées, mais cela ne sera possible que jusqu'à la fin de l'année scolaire 2013. Les filles inscrites dans le cadre de la première cohorte de Filles éveillées auront donc l'occasion d'achever deux années d'école. Le Population Council ne couvre généralement pas les frais de scolarisation individuels. Ce financement n'avait du reste pas été prévu initialement dans le cadre de Filles éveillées.

II convient de noter que même après une année d'instruction, les filles ne peuvent obtenir de certificat à l'effet que si elles peuvent produire un extrait d'acte de naissance ou une autre pièce d'identité officielle, dont la plupart ne disposent pas. II est possible d'obtenir ces documents, sous présence obligatoire d'un parent ou d'un témoin toutefois, et la demande doit souvent être faite dans le village d'origine de la fille. Les cartes d'identification produites par le programme n'étant pas soumises à vérification formelle de l'identité ou de l'âge, elles ne sont pas admises à titre officiel.

Autre difficulté encore, les filles n'ont pas pu s'inscrire aux cours du soir avant le mois de janvier et ont donc manqué un nombre considérable de classes au début de l'année scolaire. La raison en est que les cours du soir ont commencé avant les sessions de Filles éveillées et que les participantes
«Ce qui m'a vraiment fait plaisir, surtout, c'est l'inscription des filles à l'école. Aujourd'hui, elles savent lire et écrire. »

- Membre féminine de la communauté 
n'ont pas pu s'inscrire à la mi-semestre. Pour la seconde cohorte, les filles devraient être interrogées sur leur intérêt à suivre les cours du soir pendant la phase de recrutement, pour que les participantes puissent s'inscrire dès le début des classes.

Certains employeurs ont hésité à laisser leurs employées s'inscrire, ou douté qu'elles assistent régulièrement aux cours. Les VAD, de même que les visites des écoles par les mentors, les employeurs et les membres de l'Association Tié, ont dissipé les inquiétudes des employeurs et confirmé que, dans l'ensemble, les participantes étaient des élèves sérieuses. Les enseignants ont fait état d'un travail consciencieux et d'une bonne participation des filles aux classes. En général, les filles ont reçu de bonnes notes et plusieurs ont mentionné l'occasion de s'inscrire à l'école comme l'un de leurs aspects préférés de Filles éveillées. Les employeurs se sont également dits satisfaits de l'occasion offerte aux filles d'apprendre à lire et à écrire.

\subsection{Curriculum}

Dans l'ensemble, le curriculum a été qualifié de bien organisé et pertinent aux besoins des participantes. Un membre de l'Association Tié a fait remarquer en particulier qu'il appréciait la nature interactive du programme et que l'approche était utile à l'engagement des participantes. Les mentors ont dit avoir trouvé les jeux de rôles, les exemples et les histoires particulièrement utiles à l'engagement des filles. Toutes les participantes aux discussions de groupe ont pu mentionner deux ou trois choses qu'elles avaient apprises dans le cadre de leur participation au programme. Plusieurs ont mentionné savoir comment économiser de l'argent; plusieurs aussi, comment éviter la grossesse et se protéger contre les IST et plusieurs enfin, comment se laver les mains et veiller à leur propreté. Les conversations avec les mentors et les participantes ont aussi révélé plusieurs possibilités d'amélioration du curriculum, incorporées dans une révision entreprise

« Comme on le dit en Afrique, une fille instruite est une nation instruite. Cette connaissance que [les filles] vont acquérir ne se limitera pas à elles. Elle profitera à tous. Elles la transmettront à leurs sours, à leurs frères, à leurs voisins et voisines, à leurs amies et même à leurs enfants quand elles rentreront chez elles. » - Membre masculin de la communauté au troisième trimestre 2012.

\section{Pertinence du contenu}

Employeurs, mentors et participantes ont tous qualifié le contenu du curriculum de pertinent et adapté à la population ciblée. Les quatre modules ont tous été cités par différentes participantes comme étant leur préféré. Les leçons individuelles listées comme les plus utiles comprennent celles ayant trait à la grossesse, aux IST, au $\mathrm{VIH}$, à la santé reproductive, aux maladies et à l'hygiène, notamment. Les employeurs, les membres de la communauté et les participantes ont tous cité l'hygiène, à plusieurs reprises, comme particulièrement utile, et plusieurs filles ont indiqué qu'elles ne se lavaient jusque-là pas les mains après avoir été aux toilettes mais qu'elles le faisaient désormais.

"Elle a changé physiquement sur le plan de l'hygiène. J'ai remarqué qu'elle se lave les mains en sortant des toilettes. Elle est devenue très propre. Et elle partage ce qu'elle a appris sur les maladies et le VIH avec mes filles. J'en suis vraiment ravi,

d'autant plus qu'elle se montre respectueuse et qu'elle est toujours polie. » - Employeur du secteur 4 


\section{Langue}

Les sessions de Bobo-Dioulasso ont été menées en langue dioula, celles de Ouagadougou le seront en moré, car la majorité des participantes ne parlent pas le français. Les mentors ont trouvé plusieurs des concepts difficiles à expliquer en dioula. La raison en est, en partie, l'absence de mots-clés non traduits en dioula ou dont la traduction n'était pas exacte. La liste des mots-clés en dioula et en moré est le produit d'une traduction par un traducteur professionnel, dans l'alphabet respectif de chaque langue. Les mentors n'étaient cependant généralement pas elles-mêmes alphabétisées en langue dioula ou moré, le français étant la langue principale utilisée dans l'éducation formelle. Les mentors préféreraient par conséquent une liste imprimée en "franco-dioula ", selon l'alphabet français, qui leur est plus familier. Enfin, un problème s'est posé à l'impression du curriculum : dans le glossaire des mots-clés prévu en fin de document, les lettres accentuées et les symboles se sont imprimés sous forme de carrés blancs. Beaucoup des mots traduits sont donc restés illisibles pour les mentors.

\section{Alphabétisation}

Un autre problème s'est posé du fait que les filles admises au programme sont en majorité analphabètes. Le curriculum évite pour la plupart les activités qui demandent des filles qu'elles sachent lire et écrire, mais certains exercices empruntés à d'autres programmes invitent la mentor à afficher des listes, ou les filles à dessiner ou écrire alors qu'elles en sont incapables. II s'agit là d'un point principal de la révision du curriculum.

\section{Contenu}

Certaines leçons, en particulier celles concernant le cycle menstruel, la santé reproductive et la sexualité, ont été jugées trop techniques et difficiles à comprendre pour les filles. Il a été suggéré que ces leçons (en particulier les leçons 16, 17 et 19 à 22) soient simplifiées de manière à assurer la compréhension de faits essentiels (par exemple : comment éviter une grossesse et se protéger des IST ; quel est le moment où la fille risque le plus de tomber enceinte; que faire en cas d'agression sexuelle ou de grossesse non désirée). De plus, certaines des filles les plus jeunes ont eu du mal à comprendre les thèmes relatifs à la reproduction et à la sexualité. Le Module 1, sur les Compétences de vie, a été qualifié par certains de trop philosophique, difficile à traduire et difficile à comprendre pour les filles. Peu de suggestions ont cependant été émises par les mentors ou les responsables du programme quant aux révisions à apporter au contenu pour le rendre plus facile à enseigner. Le Module 4, sur les Capacités financières, a été qualifié de clair et facile à enseigner par plusieurs mentors.

\section{Soutien du curriculum}

Pour les leçons contenant une information technique sur la santé, les mentors proposent d'inviter une infirmière ou un agent sanitaire pour les aider à présenter, notamment, la session 17 (sur la reproduction et l'appareil génital masculin et féminin) et la session 15 (sur l'utilisation des services de santé par les adolescentes). La présence d'une infirmière est actuellement spécifiée pour la $15^{\mathrm{e}}$ session, mais elle n'a été assurée dans aucun des groupes.

\section{Autres thèmes d'intérêt}

Les filles ont exprimé un intérêt pour les autres thèmes suivants: le mariage forcé, les soins du nouveau-né, plus de détails sur l'accouchement et le lévirat, pratique selon laquelle une veuve est tenue d'épouser le frère ou un autre membre de la famille de son époux défunt. Le mariage forcé est le plus souvent cité comme autre thème d'intérêt. Comme indiqué plus haut, la durée de huit mois du programme a cependant été déterminée en fonction des résultats de la 
recherche formative concernant la disponibilité saisonnière des participantes. II ne serait pas réaliste d'ajouter d'autres sessions, pas plus que d'éloigner le programme de ses objectifs initiaux. Les participantes ont déclaré que toutes les sessions leur avaient paru utiles et n'ont proposé l'omission d'aucune.

\subsection{Engagement de la communauté}

\section{Engagement des employeurs}

Les employeurs et les mentors ont déclaré que les visites à domicile avaient été les plus utiles à l'assurance de leur participation et à leur encouragement à laisser leurs employées participer au programme. Les membres de la communauté ont également souligné l'importance de l'appui du programme par les leaders de la communauté, ainsi que de leur présence aux sessions communautaires.

\begin{tabular}{|} 
«Le programme touche la \\
communauté et les employeurs \\
d'abord, tandis que d'autres \\
s'adressent directement aux \\
filles. » \\
- Membre féminine de la \\
communauté
\end{tabular}

\section{Sessions communautaires}

Plusieurs sessions communautaires ont été organisées au cours du programme, dans chaque secteur. Au total, 15 sessions communautaires ont eu lieu entre octobre 2011 et juin 2012. Pour les trois secteurs originaux (2, 4 et 9), trois sessions initiales (une par secteur) ont été organisées en octobre 2011, suivies de trois autres en avril et mai, puis des trois sessions finales en juin. Pour la cohorte des secteurs entrepris plus tard (8,17 et 20), trois sessions initiales ont eu lieu en mars 2012, suivies de trois autres en juin 2012. Les sessions communautaires d'octobre ont été annoncées publiquement au moyen d'un haut-parleur monté à l'arrière d'un taxi chargé de

«Au début, mon employeur était contre. Mais le fait que je suis arrivée au bout du programme a été mon plus grand défi. Avec l'aide de ma mentor, j'ai pu convaincre mon employeur d'accepter le programme. » - Participante de 12 ans circuler dans les secteurs concernés. Des annonceurs publics et les mentors se sont également rendus de porte à porte pour en informer la population. Pour les sessions ultérieures, les leaders de la communauté se sont chargés de l'annonce. Dans le secteur 4, l'un de ces leaders a également fourni chaises, bancs et eau aux participants.

Environ 100 à 150 personnes ont assisté à chacune des sessions d'octobre ; entre 250 et 400 à chacune de celles de mars et plus de 400 à chacune des sessions de juin. On peut espérer que les nombres croissants de participants, ainsi que la contribution d'un leader de la communauté dans le secteur 4, reflètent un niveau d'engagement grandissant envers le programme.

À chaque session communautaire, le personnel de l'Association Tié et les mentors ont décrit le contenu du programme et annoncé les prochains événements (l'arrivée d'enquêteurs pour la collecte de données, par exemple), les participantes ont témoigné de l'importance du programme, les employeurs ont présenté leur point de vue et le public a été invité à poser ses questions éventuelles. Certaines sessions avaient aussi prévu un petit spectacle musical ou de dance par un groupe local. Outre les témoignages des participantes au programme et des employeurs, le Population Council avait recommandé que les filles présentent par jeux de rôles quelques-unes des compétences apprises. Ces présentations n'ont pas eu lieu. 
Contribution de la communauté Lors des discussions de groupe sur les contributions potentielles de la communauté au programme, un membre de la communauté a suggéré l'apport de bancs ainsi que d'espaces sûrs à Filles éveillées. D'autres sont cependant d'avis que le programme doit subvenir lui-même à tous ses besoins de matériel. D'autres encore estiment que la contribution de la communauté consiste simplement à laisser les filles participer au programme et à convaincre d'autres employeurs de laisser

«Les sessions communautaires ont permis à la population de voir qu'il y a des enfants, comme les employées de maison, qui ont aussi des droits que nous devons respecter. Elles ont aussi permis à certains membres de la société de voir comment le Population Council intervient à travers l'Association Tié et que les leaders sont très impliqués dans le programme, surtout les leaders religieux et traditionnels. L'association a vraiment impliqué les gens. »

- Membre masculin de la communauté

leurs employées participer aussi. Le sujet est examiné en profondeur pour la prochaine cohorte.

\section{Engagement d'anciennes participantes}

Les filles qui ont achevé le programme ont indiqué pouvoir s'imaginer dans le rôle d'assistantes aux mentors, de recruteuses d'autres filles et d'exemples à suivre dans la communauté. Elles ont proposé d'orienter les autres filles concernant le programme, dans le but d'encourager les inscriptions. Les employeurs et les mentors ont suggéré des rôles similaires pour les anciennes participantes à Filles éveillées, proposant aussi qu'elles servent d'intermédiaires entre le programme et la communauté ou au titre de représentantes du programme. Une mentor a par exemple proposé la participation de quelques filles à une annonce publicitaire du programme à la radio ou à la télévision. Un membre de la communauté a fait écho à cette suggestion, soulignant l'importance des

«Moi, je peux apprendre ce que j’ai appris à toutes les autres filles. J'ai acquis beaucoup de connaissances et je pourrais les apprendre aux filles qui n'ont pas participé au programme. » - Participante de 16 ans médias pour faire connaître le programme.

Les participantes ont exprimé leur intention de partager l'information et les compétences apprises avec leurs sœurs et amies restées au village. Elles ont également dit qu'elles parleraient du programme à leurs amies et familles, au village, et qu'elles encourageraient les futures migrantes à s'inscrire aussi. Dans le cadre d'autres programmes similaires, le Population Council a mis en œuvre un modèle de leadership en cascade, selon lequel les anciennes participantes peuvent devenir les leaders du programme. Ce modèle serait cependant particulièrement difficile dans le contexte d'une population extrêmement mobile. II n'a donc pas été intégré au plan de Filles éveillées. 


\section{RECOMMANDATIONS ET CHANGEMENTS A VENIR²}

\subsection{Mise en œuvre}

Pour le cycle 2012-2013, le feedback des associations chargées de la mise en œuvre et des mentors concernant la durée de leurs formations respectives doit être pris en compte et les formations doivent être prolongées en conséquence. Pour les organisations chargées de la mise en œuvre, la formation s'effectuera sur une période de cinq jours et pour les nouvelles mentors, elle sera de quatre jours, avec un recyclage de trois jours pour celles qui ont déjà participé à la conduite du programme.

En ce qui concerne le nombre insuffisant de VAD effectuées par certaines mentors, un nouvel outil de suivi/évaluation a été mis au point pour suivre plus efficacement le nombre de visites reçues par chaque participante au programme. De plus, les organisations chargées de la mise en œuvre ont reçu l'instruction de vérifier régulièrement la situation des VAD avec les mentors lors de leurs réunions hebdomadaires, et une référence aux VAD a été ajoutée à cet outil de suivi/évaluation. Le but de l'intervention étant, entre autres objectifs, de renforcer les capacités organisationnelles, une plus grande assistance sera offerte aux organisations chargées de la mise en œuvre en vue d'un encadrement efficace des mentors. Les organisations de mise en œuvre seront appelées à remplir les autres outils de suivi/évaluation suivants : un récapitulatif des sessions de sensibilisation de la communauté, un tableau récapitulatif des progrès des participantes inscrites aux cours du soir, un récapitulatif des réunions de travail hebdomadaires avec les mentors et deux outils relatifs à l'assistance apportées aux participantes en cas de besoin particulier (grossesse ou maladie, notamment).

\subsection{Structure du programme}

Plusieurs aspects de la structure du programme se sont très bien déroulés et peuvent continuer tels quels. Le modèle de mentors, mis en œuvre avec succès dans d'autres contextes aussi, s'est avéré fort bien adapté à celui du Burkina et il convient de le poursuivre. L'offre de nourriture aux participantes à la fin de chaque session a été jugée une partie importante du programme et doit être conservée. La programmation des sessions le dimanche après-midi doit aussi être maintenue, car il s'agit du moment qui convient le mieux aux participantes comme aux employeurs.

En guise d'appréciation de l'effort des participantes qui ont respecté leur engagement du dimanche après-midi à Filles éveillées, il a été proposé de prévoir une petite cérémonie de reconnaissance lors de la troisième et dernière session communautaire de chaque secteur en 2013. Un certificat pourra être remis aux filles qui ont participé au programme, avec un certificat de mérite aussi pour celles qui ont fait preuve d'une présence régulière (présence à au moins 20 sessions). Une attestation de participation au programme pourrait aussi aider les filles à trouver un emploi dans le futur.

\footnotetext{
2 Certains changements énoncés dans cette section avaient déjà été mis en œuvre au moment de la finalisation de cette évaluation de processus. D'autres sont planifiés ou en cours.
} 
En ce qui concerne le problème des cours du soir par opposition aux cours d'alphabétisation, une solution possible serait de trouver une autre source de financement à laquelle les filles pourraient faire appel dans le contexte d'une autre structure après leur participation à Filles éveillées. Une autre possibilité serait de n'offrir que le cours d'alphabétisation, qui peut être accompli en l'espace des huit mois du programme même si les participantes le jugent moins utile. Moins de participantes s'y inscriraient peut-être et le cours pourrait s'avérer moins avantageux pour celles qui s'y inscrivent. Une troisième possibilité, moins désirable, serait de n'offrir aucune forme d'aide financière à l'éducation. II est recommandé, pour la prochaine cohorte, de proposer l'inscription aux cours du soir, en indiquant clairement aux participantes que cette assistance ne sera pas offerte au-delà de la conclusion du programme Filles éveillées. Les partenaires chargés de la mise en œuvre devront plutôt rechercher l'aide d'autres organismes aptes à assurer le financement continu de l'éducation des filles désireuses de poursuivre leurs études. Par exemple, l'association ADEP offre elle-même cette assistance financière à certaines élèves. Pour faciliter leur avancement et l'obtention de certificats, les participantes doivent être guidées et encouragées par les mentors à obtenir les documents officiels requis avec l'aide de leur famille lors de leur retour au village. Un bref exercice expliquant l'importance de ces documents officiels et la manière de les obtenir a été ajouté à la première session du curriculum. Les mentors seront aussi appelées à apporter l'aide possible.

Après discussions avec les mentors, l'Association Tié et le Population Council, il a été décidé que les tranches d'âge resteront identiques (11 à 16 ans à Bobo-Dioulasso et 15 à 18 ans à Ouagadougou) et que les groupes de Bobo ne seront pas subdivisés en fonction de l'âge. Si certaines des cadettes ont éprouvé des difficultés à comprendre certaines leçons, le problème n'a pas été résolu par leur séparation dans un groupe distinct. II conviendrait plutôt de réviser le curriculum pour en accroître l'accessibilité aux filles à tous les âges de la tranche considérée.

\subsection{Curriculum}

Le feedback recueilli pour l'évaluation de processus a permis l'apport de révisions ciblées significatives au curriculum, en termes de contenu, d'organisation et de ressources supplémentaires durant le troisième trimestre 2012. Le principal souci exprimé par les mentors et les partenaires chargés de la mise en œuvre concernait l'adaptation du curriculum à la population ciblée, en tenant compte de l'âge et de l'analphabétisme des filles. Tous les exercices requérant lecture ou écriture ont été modifiés pour répondre aux besoins des participantes analphabètes. Le curriculum ne comprend plus aucun exercice demandant aux participantes de lire ou d'écrire elles-mêmes et il est rappelé aux mentors de lire systématiquement à haute voix le texte écrit sur les tableaux affichés. Pour les exercices des sessions 29 et 30, des options de remplacement sont également proposées au cas où les activités simplifiées seraient toujours trop compliquées. De nouvelles ressources visuelles ont été ajoutées aux sessions 18 et 23 et le curriculum prévoit désormais la présentation d'échantillons réels de différentes formes de contraception à la session 24. Il a été déterminé que la session 13 contenait trop d'informations pour que les filles puissent les retenir. La quantité d'informations a donc été réduite et l'information en soi, simplifiée.

De plus, certains exercices ont été modifiés pour faciliter la compréhension des cadettes non encore sexuellement actives ou même pubères, en particulier concernant les sessions 22 et 23. 
Ces deux sessions ont aussi été réorganisées pour donner aux filles une compréhension élémentaire de la puberté et des organes génitaux avant de passer à la menstruation, à l'ovulation et à la reproduction. On peut espérer que cette approche plus visuelle, présentée dans un langage plus simple, avec moins d'informations et plus d'exemples, facilitera la compréhension. Comme l'ont aussi proposé les mentors, le temps réservé à ces leçons a été porté à 90 minutes. Des vignettes et des exemples ont par ailleurs été ajoutés aux sessions 6 et 7 pour mieux illustrer les idées abstraites telles que les valeurs et l'affirmation de soi.

Le module consacré aux compétences financières aide les participantes à apprendre comment économiser leur argent et à se fixer des buts d'épargne. Pour en renforcer l'impact, ce module a été déplacé et est maintenant le deuxième présenté plutôt que le dernier. Les filles peuvent ainsi être encouragées à pratiquer ce qu'elles ont appris sur l'épargne tout au long du programme et les mentors peuvent suivre leurs progrès. Un outil de suivi/évaluation destiné à suivre les buts d'épargne des participantes a également été mis au point. Concernant l'usage d'argent véritable par opposition aux faux billets pour les exercices, l'organisation responsable du programme devrait fournir cet argent aux mentors, sur les fonds obtenus du Population Council. Comme les mentors peuvent emprunter l'argent, l'utiliser pour les exercices et le retourner ensuite à l'organisation, il n'est pas nécessaire de budgétiser de ressources supplémentaires pour la mise en œuvre de ce module.

Bien que la contrainte de 30 semaines interdise l'ajout de sessions supplémentaires, certains exercices ont été ajoutés à celles qui étaient déjà courtes et simples. Par exemple, étant donné le succès et la simplicité relative de la session sur l'hygiène corporelle, une activité a été ajoutée sur l'hygiène dentaire : elle pourra avoir un impact significatif sur l'alimentation et la santé des filles, de même que sur celle de leurs enfants déjà nés ou à venir.

Sur le plan de la langue, le problème d'impression et des mots illisibles en dioula et en moré a été résolu. Une ressource complémentaire, rédigée en " franco-dioula " et en "franco-moré " pour faciliter la prononciation, devra être fournie aux mentors.

Au moins une visite par une infirmière était suggérée dans le curriculum précédent, mais aucun groupe n'en a bénéficié. II est proposé que l'organisation chargée de la mise en œuvre prenne les dispositions nécessaires à ce sujet plusieurs semaines à l'avance et, au besoin, qu'elle prévoie une deuxième infirmière ou une session de remplacement pour assurer que les participantes reçoivent l'information dont elles ont besoin. D'autres rappels et détails de préparation ont été ajoutés dans le but d'encourager les démarches de planification appropriées. Le Population Council tiendra les organisations chargées de la mise en œuvre responsables de l'organisation effective de ces visites. Une autre suggestion serait d'inclure une visite de groupe dans un centre de santé, pour que les filles puissent aussi se familiariser avec l'endroit. Dans le secteur 8, les participantes ont exprimé un intérêt pour le dépistage du VIH et demandé l'aide de leur mentor à ce sujet. L'organisation d'une visite de groupe pourrait soulager l'appréhension individuelle des filles et les encourager à recourir aux services disponibles, pour le dépistage du VIH ou à d'autres fins. Une activité impliquant la visite d'une personne représentant une banque locale a aussi été ajoutée à la session 13. Cette visite exigera de même une démarche de planification à l'avance. 


\subsection{Engagement de la communauté}

Les efforts d'engagement de la communauté se sont généralement révélés très positifs. Les visites à domicile et les sessions communautaires ont été largement appréciées et doivent se poursuivre. II convient de continuer à aborder les leaders de la communauté et à les encourager à contribuer au programme dans la mesure de leurs capacités, qu'il s'agisse de l'apport d'un espace sûr, de bancs durant les rencontres ou de sièges ou de rafraîchissements à l'occasion des sessions communautaires. L'exemple des bancs et de l'eau apportés par un leader du secteur 4 pourrait être évoqué pour en inspirer d'autres peut-être à soutenir plus activement le programme. L'Association Tié a rencontré de nombreux leaders communautaires durant le troisième trimestre 2012, afin de discuter leur contribution possible au programme. Pour rendre les sessions communautaires plus interactives et plus engageantes, les filles pourraient interpréter de petits jeux de rôles et montrer ainsi ce qu'elles ont appris. II a par ailleurs été décidé qu'à Bobo-Dioulasso, l'Association Tié et les mentors vont organiser de petits groupes de discussion avec les employeurs dans le but d'obtenir leur soutien et de renforcer les relations entre les employeurs et le programme Filles éveillées.

Pour les anciennes participantes, le Population Council envisage la mise au point d'une ressource de supports visuels pertinents et l'appui des partenaires de mise en œuvre concernant la formation des participantes disposées à transmettre leurs connaissances à leurs pairs et à d'autres membres de leurs communautés et villages. L'élaboration d'une ressource utile aux anciennes participantes devra tenir compte du fait que la plupart des participantes aux programmes sont analphabètes.

\section{CONCLUSION}

Les observations tirées de la collecte de données quantitatives et qualitatives ont révélé le succès considérable du programme Filles éveillées : il est largement apprécié et a été bien reçu par les participantes, les mentors, les membres de la communauté et tous les autres intéressés. En sa qualité de programme pilote, les obstacles à une mise en œuvre sans incidents auraient été inévitables. Par leur détermination et leur dynamisme, l'Association Tié, le Population Council et la Jacobs Foundation ont cependant permis l'accès au nombre de filles ciblé et la mise en œuvre du programme telle qu'elle avait été planifiée. Cette première cohorte a permis de tester le modèle programmatique en vue de son amélioration. Plusieurs ajustements ont été apportés en conséquence. L'évaluation d'impact prévue en 2013 révélera la mesure dans laquelle le programme aura réussi à atteindre ses objectifs de réduction de la vulnérabilité et d'élargissement des horizons de cette population particulièrement vulnérable. Notre espoir est que les résultats de l'évaluation de processus et les ajustements correspondants apportés au programme permettront de réaliser ces objectifs. 


\section{REFERENCES}

Population Council. (2012). Burkina Faso: Overview. Obtenu le 22 juillet 2012 sur

Population Council : http://popcouncil.org/countries/burkinafaso.asp

Population Council. (2012). Developing Capacity for Targeted, Evidence-based

Programming and Asset Building for Adolescent Girls. Obtenu le 28 septembre 2012 sur Population Council :

http://popcouncil.org/projects/164_DevCapProgramAdolGirls.asp\#/jQueryUITabs1-1

Population Council. (2009). The Adolescent Experience In-Depth: Using Data to Identify and Reach the Most Vulnerable Young People: Burkina Faso 2003. New York : Population Council.

Population Council. (2012). Who We Are: Overview. Obtenu le 22 juillet 2012 sur Population Council : http://popcouncil.org/who/index.asp

Population Council; Guttmacher Institute. (2010, June 14). Atteindre les Adolescents Qui ont les Plus Grands Besoins: Le Cas du Burkina Faso. Ouagadougou, Burkina Faso.

Population Reference Bureau. (2011). World Population Data Sheet. Obtenu le 22 juillet 2012 sur Population Reference Bureau : http://www.prb.org/pdf11/2011population-datasheet_eng.pdf

United Nations Development Programme. (2011). Burkina Faso Country Profile. Obtenu le 10 août 2012 sur International Human Development Indicators : http://hdrstats.undp.org/en/countries/profiles/BFA.html 


\section{Annexe 1: Outils de collecte de données qualitatives}

\subsection{Guide de Discussion de Groupe avec les Mentors}

Bonjour, et merci pour avoir accepté de participer à la discussion de groupe avec les autres mentors pour le programme «Filles Éveillées ». Je suis et je suis ici pour apprendre un peu plus sur ce que vous pensez sur le programme et comment on pourrait l'améliorer. On voudrait avoir toutes vos idées et aussi vos critiques positives ou négatives. N`hésitez pas à me dire aussi les choses négatives parce qu'on veut savoir aussi ce qui n’a pas marché pour améliorer lors de la deuxième phase du programme.

1. Est-ce que chacune de vous peut nous donner son nom et une chose que vous aimez plus dans le rôle du mentor?

Questions sur la formation (mise en ouvre du programme) : On va commencer avec des questions sur votre formation. Avant de commencer le programme, vous avez participé à une formation de trois jours animée par les chargés de programme de l'Association Tié. Il y a 4 domaines ciblés par le programme «Filles Éveillées »: les compétences de vie courante, la santé et l'hygiène, la santé de la reproduction, et les capacités financières.

2. Qu'est-ce que vous pensez de la durée, la structure, le contenu, les approches utilisées par les formateurs pour le transfert des connaissances et la qualité de la formation ? Si c'est à refaire, qu'est-ce que vous ajouteriez à la formation pour vous permettre de mieux comprendre les objectifs globaux du projet?

3. Dans quel module êtes-vous le plus à l'aise et le plus préparée à enseigner ? Dans quel module êtes-vous le moins à l'aise et avez-vous le plus de difficultés à enseigner?

4. Qu'est-ce que vous ajouteriez à la formation pour vous permettre de mieux comprendre et enseigner les 4 domaines ?

5. Comment est-ce que le soutien d'Association Tié vous aidait à répondre aux défis rencontrés tout au long du programme ? Quel type de soutien était le plus utile pour vous?

6. Quelles suggestions précises faites-vous pour améliorer la formation des mentors et/ou le soutien des mentors par les chargés de programme de Tié tout au long du programme?

Contenu : Maintenant je vais vous poser des questions sur le curriculum du programme.

7. Est-ce que les exercices, les histoires de vie et les jeux de rôle utilisés dans le curriculum sont adaptés à la réalité des filles dans le programme ? Quelles stratégies vous a semblé la plus efficace pour engager/intéresser les filles à la session ?

8. Qu'est-ce que vous pensez de la pertinence des quatre thèmes abordés par le programme pour la tranche d'âge ciblée dans « Filles Éveillées » (11-16 ans)? Est-ce que les filles ont manifesté l'intérêt pour d'autres thèmes? Lesquels ? Et qu'est-ce qu'elles souhaiteraient exactement connaitre en plus des thèmes qui sont déjà abordés dans le curriculum?

Structure : Maintenant je vais vous poser des questions sur la structure du programme.

9. Qu'est-ce que vous avez pensé de la structure d'utiliser un espace sûr, d'avoir des amies filles, et un mentor? Quels sont les avantages de cette structure? Quels sont les inconvénients? 
10. Comment cela se distingue des approches d'autres programmes qui ciblent aussi les employées de maison?

11. Qu'est-ce qui fera le guide des mentors plus complet? (Considérez le niveau d'instruction pour la population ciblée, la variété des activités, les mots clés en fin du curriculum, les visites des personnes ressources dans la communauté, et les outils pour les mentors). Merci de signaler 2-3 choses dans le guide du mentor ou même de la structure du curriculum que vous souhaiterez changer avant de lancer ce programme pour la seconde cohorte.

12. Qu'est-ce que vous avez pensé de la durée de huit mois, une fois par semaine, et d'environ 90 minutes pour chaque session? Avez-vous eu suffisamment de temps pour animer les sessions et engager les filles?

13. Certaines d'entre vous ont travaillé avec des filles très jeunes (11-13 ans), d'autres avec des filles plus âgées (14-16 ans) et d'autres encore avec des filles dont l'intervalle d’âge est plus grand (11 à 16 ans).. Quels étaient les avantages et les inconvénients/difficultés liés à chaque modèle ?

14. Est-ce que les employeurs semblent être engagés pour le programme ? Est-ce qu'ils ont suivi le programme et ils parlent souvent avec les filles sur ce qu'elles apprennent lors des sessions?

15. Qu'est-ce qu'on peut faire pour rendre les visites à domiciles plus utiles afin de gagner le soutien des employeurs et aborder les problèmes rencontrés par les filles de façon plus efficace?

16. Quelle était le plus grand défi pour vous dans le programme «filles éveillées »?

\section{Future}

17. Quel rôle voyez-vous pour les filles qui ont déjà terminé le programme et qui seront à Bobo lors de la deuxième phase? Par exemple, comme assistant au mentor ? Recruteuse ? Intermédiaire avec la communauté ?

18. Quel rôle voyez-vous pour les filles qui ont déjà terminé le programme et qui vont rester dans leur village d'origine définitivement?

19. Selon vous comment le programme pourrait engager davantage les employeurs et les communautés?

\section{Fin}

20. Quelques autres commentaires ou suggestions avez-vous? 


\subsection{Guide de Discussion de Groupe pour les Chargés de Programme au Niveau de Tié}

Bonjour, et merci pour avoir accepté de participer à la discussion de groupe avec les autres personnes chargés du programme «Filles Éveillées». Je suis et je suis ici pour apprendre un peu plus sur ce que vous pensez sur le programme et comment on pourrait l'améliorer. On voudrait avoir toutes vos idées et aussi vos critiques positives ou négatives. $\mathrm{N}$ ‘hésitez pas à me dire aussi les choses négatives parce qu'on veut savoir aussi ce qui n’a pas marché pour améliorer lors de la deuxième phase du programme.

1. Est-ce que chacun de vous peut nous dire son nom et son rôle dans l'Association Tié et dans le programme «Filles Éveillées »? Qu'est-ce que chacun de vous fait exactement dans le programme?

Questions sur la mise en œuvre du programme : Avant de commencer le programme "Filles Éveillées », vous avez reçu une formation de 4 jours de Population Council en septembre 2011 sur les objectifs et la structure du programme, le lancement et les outils de suivi-évaluation, etc., et tout au long du programme vous avez reçu également leur feedback et soutien soit à travers les conversations hebdomadaires ou les visites de terrain.

2. Qu'est-ce que vous pensez de la durée, la structure, et la qualité de la formation ? Qu'estce que vous ajouteriez à la formation pour vous permettre de mieux comprendre les objectifs globaux du projet ou les attentes précises de votre rôle dans le projet?

3. Quelles sont 2 ou 3 choses que vous avez apprises au cours de cette formation? Quelle était la chose la plus utile pour vous?

4. Vous avez reçu du feedback du Council tout au long du programme (à travers des visites sur le terrain, les appels hebdomadaires, le feedback sur les documents écrits). Quel type de feedback était le plus utile ? Comment est-ce que le Council pourrait mieux soutenir l'Association Tié, ou collaborer avec vous pour avoir plus d'impacts?

5. En général, quel exploitation faites-vous des outils de suivi-évaluation remplis ? Comment vous avez utilisé l'information que vous avez reçue sur les outils de suivi évaluation pour surveiller les progrès du programme et réajuster des choses en fonction des données? Citer 2 ou 3 exemples.

6. Quel outil était le plus utile ? Quel outil était le moins utile? Pensez-vous que vous avez les outils nécessaires pour renseigner le programme ou bien il y a des outils qui manquent et qu'il serait nécessaire de les développer ? Si oui, pour renseigner quelle activité ?

7. Qu'est-ce que vous pensez des canevas des rapports narratifs que vous remplissez? Pensez-vous qu'on doit changer le format? Si oui, comment?

8. Quels types de soutien apportez-vous aux mentors? (Demandez-leur les comptes rendus de réunion avec les mentors pour apprécier le contenu.)

9. Quels types de soutien que les mentors ont cherché auprès de l'Association Tié? Vous vous sentiez bien préparés pour répondre à leurs questions?

\section{Structure}

10. Qu'est-ce que vous pensez de la structure d'utiliser un espace sur, un groupe de filles, et un mentor? Quels sont les avantages de cette structure? Quels sont les inconvénients? 
Comment cela se distingue des approches d'autres programmes ciblant les filles domestiques?

11. Qu'est-ce que vous pensez de la durée de huit mois, une fois par semaine, et d'environ 90 minutes pour chaque session?

12. Après vos visites au programme et vos discussions avec les mentors, qu'est-ce qu'on pourrait faire pour améliorer l'approche de «Filles Éveillées» pour mieux aborder les défis que les filles migrantes rencontrent?

\section{Contenu}

13. Qu'est-ce que vous pensez de l'alphabétisation/cours du soir des filles? Considérez l'utilité, le suivi de ses filles, les résultats atteints, etc. et comment faire pour améliorer ce volet?

14. Quel était le plus grand défi que vous avez dans le programme « Filles éveillées »?

\section{Future}

15. Quel était la relation avec les autorités locales, les leaders communautaires ou la communauté ? Quelle pourrait être leur contribution pour le maintien des espaces sûrs ? D'autres contributions?

16. Association Tié a exprimé son intérêt de répondre aux questions des filles qui sont victimes de violence, de grossesse non désirée, d'insécurité, ou qui ont d'autres problèmes chez leurs employeurs. Quelles sont 2 ou 3 idées pour répondre à ces différentes situations pour un plus grand effet? Comment comptez-vous de façon pratique y procéder lorsque vous avez affaire à des cas concrets?

17. Quel rôle voyez-vous pour les filles qui ont déjà achevé le programme et qui vont revenir à Bobo après la saison des pluies? Par exemple, comme assistant au mentor ? Recruteuse ? Intermédiaire avec la communauté ?

18. Quel rôle voyez-vous pour les filles qui ont déjà achevé le programme et qui vont rester dans leur village d'origine définitivement?

19. Selon vous, comment le programme pourrait engager davantage les employeurs et les communautés? 


\subsection{Guide d'Entretien pour Membres de la Communauté}

Bonjour, et merci pour avoir accepté de participer à la discussion de groupe avec les autres membres de la communauté. Je suis et je suis ici pour apprendre un peu plus sur ce que vous pensez sur le programme « Filles éveillées » et comment on pourrait l’améliorer.

En rappel, «Filles Éveillées » est un programme pour les jeunes filles migrées qui travaillent comme employées de maison. Durant huit (8) mois, les filles participent à une session chaque dimanche avec d'autres filles de leur âge et un mentor. Le programme cherche à développer des compétences chez les filles dans quatre domaines : les compétences de vie courante, la santé et l'hygiène, la santé de la reproduction, et les capacités financières.

On voudrait avoir toutes vos idées et aussi vos critiques positives ou négatives. N‘hésitez pas à me dire aussi les choses négatives parce qu'on veut savoir aussi ce qui n’a pas marché pour améliorer lors de la deuxième phase du programme.

1. Est-ce que chacun de vous peut se présenter?

\section{Mise en ouvre /contenu}

2. Comment est-ce que vous avez connu ce programme pour la première fois? Comment estce que vous avez été informé de « Filles Éveillées » tout au long du programme?

3. Pouvez-vous nous dire brièvement ce que le programme fait? Comment le programme «Filles Éveillées » se distingue des autres programmes qui ciblent les filles employées de maison?

4. Selon vous, comment est-ce que ce programme pourrait enrichir davantage les filles/être profitable aux filles? Et la communauté?

5. Pour gagner le maximum de soutien de la communauté, comment est-ce qu'on peut ajuster nos principaux messages à la communauté?

6. Quelle pourrait être la contribution de la communauté pour le programme «Filles Éveillées »? (Par exemple, une espace sur, d'alimentation pour les sessions, bancs pour chaque espace, contribution en espèces, etc.)

7. En plus des sessions communautaires, qu'est-ce qu'on peut faire pour renforcer l'engagement de la communauté dans le programme?

\section{Future}

8. Quel rôle voyez-vous pour les filles qui ont déjà achevé le programme? Par exemple, comme assistant au mentor ? Recruteuse ? Intermédiaire avec la communauté ? Comment est-ce que les filles peuvent partager ses compétences et ses connaissances avec d'autres filles dans la communauté ?

9. Quelques autres commentaires ou suggestions avez-vous? 


\subsection{Guide de Discussion de Groupe pour Filles qui ont Terminé le Programme}

Bonjour, et merci pour avoir accepté de participer à la discussion de groupe avec les autres filles qui ont complétées le programme «Filles Éveillées ». Je suis et je suis ici pour apprendre un peu plus sur ce que vous pensez sur le programme et comment on pourrait l'améliorer. On voudrait avoir toutes vos idées et aussi vos critiques positives ou négatives. $\mathrm{N}$ ‘hésitez pas à me dire aussi les choses négatives parce qu'on veut savoir aussi ce qui n’a pas marché pour améliorer lors de la deuxième phase du programme.

1. Est-ce que chacun de vous peut se présenter, et dire une chose que vous avez aimé dans le programme « Filles Éveillées »?

\section{Contenu}

2. Est-ce que les thèmes abordés par le programme étaient pertinents pour vous et utiles dans votre vie ? Quels sont 2 ou 3 choses que vous savez faire maintenant et que vous ne saviez pas faire avant de participer au programme?

3. Quelles sont les deux sessions ou sujets qui étaient les plus utiles pour vous ? Quelles sont les deux sessions ou thèmes qui étaient les moins utiles pour vous? Pourquoi ?

4. Quel sont les thèmes qui n'ont pas été couverts par le programme et qu'on pourrait abordés s'il y avait une suite? Et pourquoi ?

\section{Mise en ouvre du programme}

5. Est-ce que le programme « Filles Éveillées » contribue à répondre à vos besoins ? Qu'estce que l'on pourrait faire pour mieux répondre à vos besoins ?

6. Qu'est-ce que vous pensez sur les visites à domiciles ? Est-ce qu'elles étaient utiles pour vous et pour vos employeurs? Pourquoi ?

7. Qu'est-ce que vos employeurs pensent du programme ? Est-ce qu'elles soutiennent votre participation? Pourquoi ? Est-ce que vos relations avec vos employeurs ont changé de quelque manière à cause du programme ?

8. Est-ce que vous pouvez me décrire votre relation avec vos mentors ? Est-ce que vous lui demandiez des conseils ou bien vous avez confiance que vous pouvez lui demander des conseils en cas de besoins ou vous confier à elle? Vous vous sentiez à l'aise avec elle? Selon vous, quelles qualités sont les plus importants chez un mentor ?

9. Vous vous sentais à l'aise avec les autres filles de votre groupe ? Pourquoi ? Est-ce que l'âge des autres filles est un facteur important pour vous pour se sentir à l'aise?

\section{Structure}

10. Qu'est-ce que vous pensez de la durée du programme ? (Pour celles qui ont fait 4 mois, demandez-leur si elles sont prêtes à participer à 8 mois de programme.) Que pensez-vous de la durée et la fréquence des sessions ? (une fois par semaine et si c'est deux fois dans la semaine ?). Le temps était-il suffisant pour comprendre le contenu de la session ?

11. Selon vous, comment est-ce que vous pouvez contribuer à appuyer les filles de la $2^{\text {ème }}$ cohorte du programme ? Expliquer quel pourrait être votre rôle (à Bobo ou au retour au village). Est-ce que vous pensez que vous allez partager les informations que vous avez 
apprises aux filles lorsque vous retournera dans votre village? Comment allez-vous procéder?

12. Quel était le plus grande défi pour vous dans le programme « Filles Éveillées »?

13. Est-ce que vous recommanderiez ce programme aux autres filles comme vous ? Pourquoi?

\section{Fin}

14. Avez-vous d'autres commentaires ou observations? 


\subsection{Guide d'Entretien pour les Employeurs}

Bonjour, et merci pour avoir accepté de participer à un entretien avec moi. Je suis et je suis ici pour apprendre un peu plus sur ce que vous pensez sur le programme «Filles Éveillées » et comment on pourrait l’améliorer.

En rappel, «Filles Éveillées » est un programme pour les jeunes filles migrées qui travaillent comme employées de maison. Durant huit (8) mois, les filles participent à une session chaque dimanche avec d'autres filles de leur âge et un mentor. Le programme cherche à développer des compétences chez les filles dans quatre domaines : les compétences de vie courante, la santé et l'hygiène, la santé de la reproduction, et les capacités financières.

On voudrait avoir toutes vos idées et aussi vos critiques positives ou négatives. N‘hésitez pas à me dire aussi les choses négatives parce qu'on veut savoir aussi ce qui n’a pas marché pour améliorer lors de la deuxième phase du programme.

\section{Contenu}

1. Pouvez-vous nous dire brièvement ce que le programme «Filles Éveillées » fait ? Qu'estce qui vous a le plus plu dans le programme « Filles Éveillées » ?

2. Comment le programme «Filles Éveillées» se distingue des autres programmes qui ciblent les filles employées de maison?

3. Est-ce que vous pensez que votre employée a profité de ce programme ? De quelle manière ? Comment le savez-vous ?

4. En tant qu'employeur, avez-vous profité de ce programme? Pouvez-vous nous donner quelques exemples concrets ? Est-ce que votre employée a souvent raconté à la famille ce qu'elle a appris aux sessions?

5. Qu'est-ce qui ne vous a pas plu dans le programme «Filles Éveillées » et mérite d'être améliorer? Quelles propositions faites-vous pour l'améliorer?

\section{Mise en ouvre du programme}

6. Est-ce que vous pensez que le programme "Filles Éveillées" est une approche appropriée/un programme pertinent pour aborder les défis que les filles migrantes rencontrent ? Qu'est-ce que l'on pourrait faire pour l'améliorer?

7. Qu'est-ce que vous pensez des visites à domicile et qu'est-ce que vous avez discuté avec le mentor lors de ces visites? Est-ce que les visites à domicile étaient utiles ?

8. Est-ce que vous avez participé à quelques sessions communautaires du programme ? Qu'est-ce que vous en pensez? Que peut-on améliorer dans ces sessions communautaires?

\section{Structure}

9. Il y a 8 mois de cela (ou 4 mois pour les nouveaux groupes), lorsque les mentors sont venues vous expliquer le programme, vous avez donné votre accord à ce que votre employée participe au programme. Quelles sont les difficultés que vous avez rencontrez 
par la suite qui ont empêché votre employée de venir régulièrement aux sessions les dimanches?

\section{Pour terminer}

10. Est-ce que vous recommanderiez ce programme aux autres employeurs et aux autres employées ? Pourquoi/pourquoi pas ? Est-ce que vous laisserez ou encouragerez votre future employée à y participer ?

11. Avez-vous d'autres commentaires ou suggestions? 


\section{Annexe 2: Outils de suivi et d'évaluation ${ }^{3}$}

\subsection{Canevas d'Appréciation des Sessions}

Ce canevas doit être renseigné par la mentor à chaque session

Date :

Ville/Site/espace sûr :

Nom de l'association :

Nom du mentor :

Nom du groupe :

Session abordée (numéro et titre) :

Utilité de la session pour les filles :

Utile

Moyennement utile |

Pas utile

Appréciation de la durée de la session :

Longue

Acceptable

Trop courte

Pertinence des exercices de la session :

Pertinent

Assez pertinent $\mid$

Pas pertinent

Compréhension des exercices de la session par les filles :

Bonne compréhension

Compréhension moyenne

Pas compréhensible

Compréhension/clarté de la session :

Bonne compréhension par les filles

Compréhension moyenne

Compréhension difficile

Difficultés rencontrées par le mentor dans l'animation de la session :

Suggestions de solutions:

\footnotetext{
${ }^{3}$ Les outils de suivi/évaluation présentés dans cette annexe sont les versions utilisées de 2011 à 2012. Les versions mises à jour ont été soumises aux organisations chargées de la mise en œuvre. Les versions finales seront remises au donateur dès leur finalisation.
} 
Aspects/points de la session à améliorer :-

Suggestions globale sur la session :

Signature du mentor : 


\subsection{Tableau de Présence des Filles aux Sessions}

Date :

-Ville/Site/espace sûr

Secteur/quartier :

-Nom de l'association :

Nom du mentor :

-Nom du groupe :

NB : Cette fiche est conçue pour chaque groupe et les noms des filles peuvent être préalablement notés. A chaque session, il vous suffit de cocher la case correspondante lorsque la fille est présente. Cette fiche doit être renseignée par le mentor.

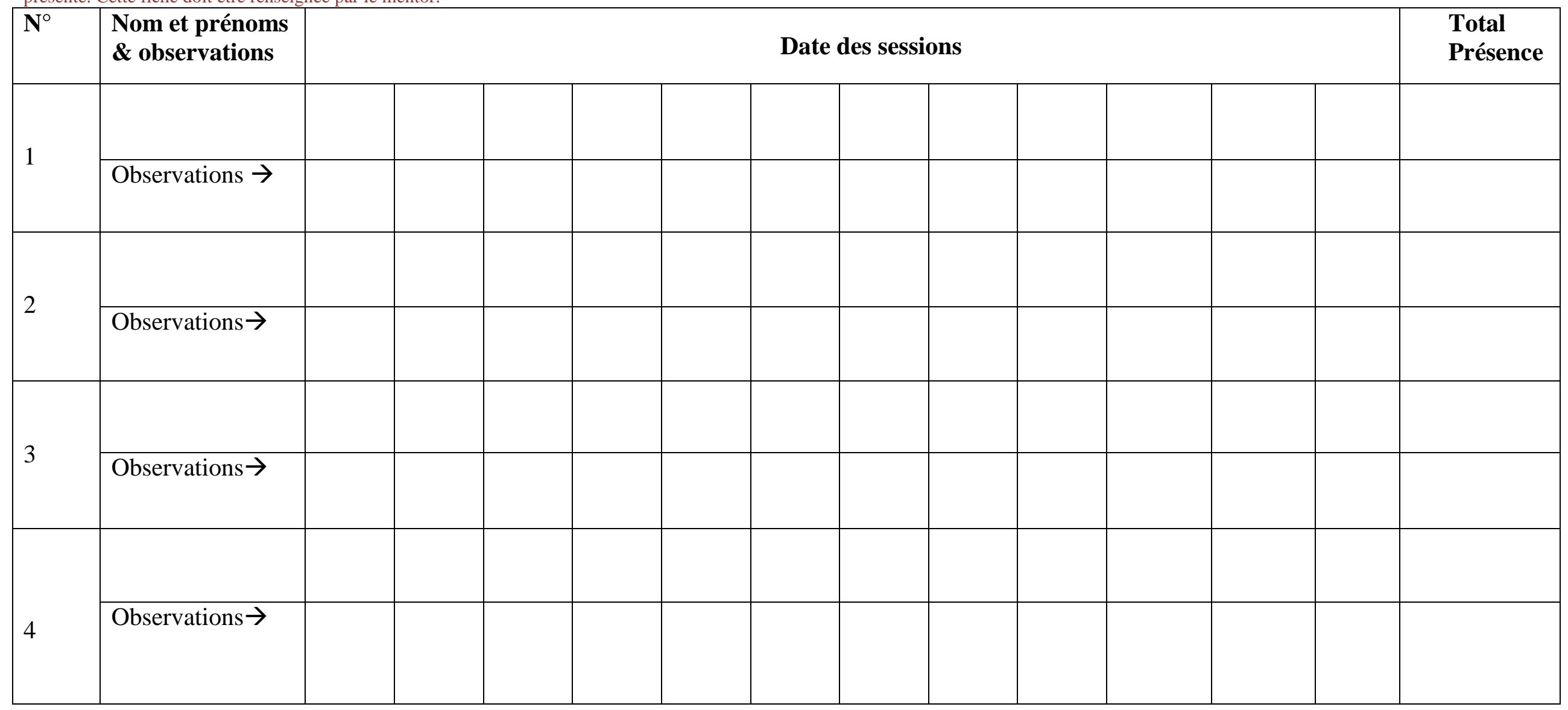




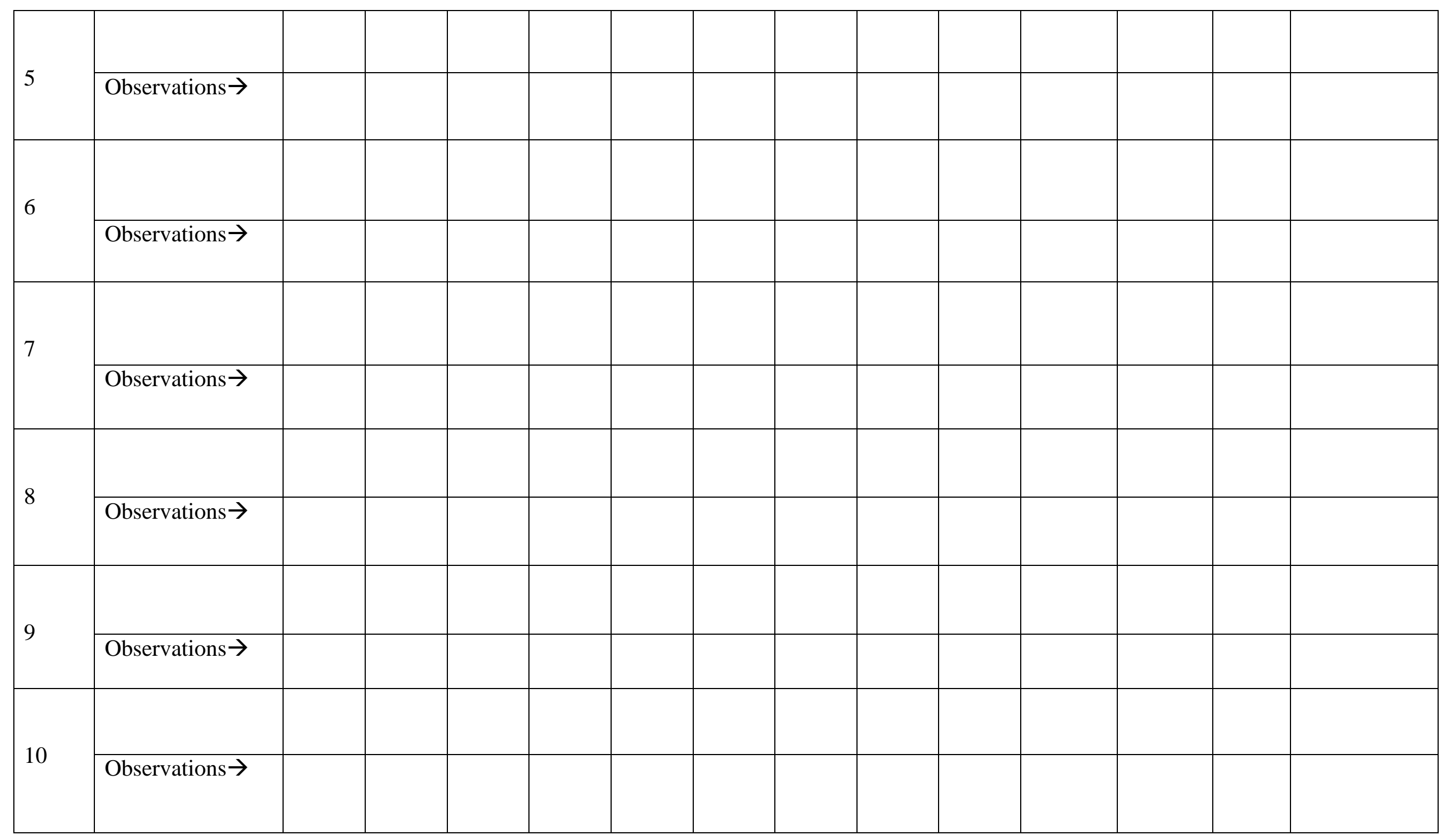




\subsection{Fiche Individuelle des Visites à Domicile}

Cette fiche doit être renseignée à chaque fois que le mentor effectue une visite d’une fille à domicile.

Date :

Ville/Site/espace sûr :

Secteur/quartier :

Adresses du domicile de la fille :

Nom de l'association :

Nom du mentor :

Nom du groupe :

Nom et prénom de la fille :

Age de la fille :

Qui était présent(e) (fille seule, avec employeur, ...) :

Objet de la visite :

Contenu de la visite :

Compte rendu narratif (expliquer comment s'est passée la visite, les résultats constatés, etc.).

Date probable de la prochaine visite :

Motif de la prochaine visite :

Signature : 


\subsection{Fiche de Supervision des Activités des Mentors}

Cette fiche doit être renseignée par le chargé de programme ou toute autre personne de l'association qui effectue une supervision du travail du mentor.

Date :

Ville/Site :

Secteur/quartier :

Nom de l'association :

Nom du mentor :

Nom du groupe :

Nom du superviseur :

Objectifs de la supervision :

Thème de la session :

Constats:

\section{Appréciation du déroulement de la session}

Par rapport au mentor:

Par rapport à la participation du groupe:

Par rapport aux approches utilisées:

Points forts:

Points à améliorer:

Signature du superviseur : 


\subsection{Canevas de Rapport Narratif des Associations}

Ce canevas est proposé pour le rapport trimestriel des associations

\section{Page de garde}

Date :

Ville/Site :

Secteur/quartier :

Nom de l'association :

Période du rapport :

\section{Partie narrative}

Contexte/Intro

Objectifs

Résultats Attendus

Description et analyse des activités réalisées (mettre l'accent sur l'intérêt des participantes par rapport au programme, les faits marquants, le degré de participation des filles au programme, qu'est ce qui a marché, qu'est-ce qui mérite d'être améliorer, ............)

Difficultés rencontrées

Leçons apprises

Suggestions et Recommandations

\section{Leçons apprises (Fiche d'orientation)}

\begin{tabular}{|c|l|l|}
\hline Domaines et sujets & Constats & Leçons apprises \\
\hline $\begin{array}{l}\text { Qu'avez-vous appris dans le travail avec } \\
\text { les filles employées de maison? }\end{array}$ & & \\
\hline Avec les Employeurs & & \\
\hline Avec les Mentors & & \\
\hline $\begin{array}{l}\text { Dans l'approche de passer par des mentors } \\
\text { pour mettre en œuvre le programme }\end{array}$ & & \\
\hline Dans la Mobilisation des communautés & & \\
\hline $\begin{array}{l}\text { Dans la Mobilisation des filles du } \\
\text { programme }\end{array}$ & & \\
\hline Par rapport au contenu du programme & & \\
\hline $\begin{array}{l}\text { Par rapport aux approches utilisées pour les } \\
\text { sessions }\end{array}$ & & \\
\hline $\begin{array}{l}\text { Par rapport à l'approche de travailler avec } \\
\text { des partenaires }\end{array}$ & & \\
\hline Etc. & & \\
\hline
\end{tabular}




\subsection{Canevas du Rapport des Enseignants sur l'Encadrement des Filles du Programme}

Ce canevas doit être renseigné par l'enseignant tous les 2 mois.

Période du rapport :

Secteur :

Nom de l'enseignant :

Établissement :

Nombre de filles inscrites :

Nombre de filles qui viennent régulièrement ( $90 \%$ ou plus) :

Classes couvertes : CP1 ( ) CP2 ( ) CE1 ( ) CE2 ( ) CM1 ( ) CM2 ( ) 6 ème ( )

Appréciation de la participation des filles (l'assiduité et participation aux cours, la motivation...)

Progrès fait durant la période :

Difficultés rencontrées :

Suggestions et recommandations :

Signature de l'enseignant

Visa Association TIÉ 